\title{
24. PLIOCENE-PLEISTOCENE FLUCTUATIONS IN COMPOSITION AND ACCUMULATION RATES OF EOLO-MARINE SEDIMENTS AT SITE 798 (OKI RIDGE, SEA OF JAPAN) AND CLIMATIC CHANGE: PRELIMINARY RESULTS ${ }^{1}$
}

\author{
M. Dersch ${ }^{2}$ and R. Stein ${ }^{2}$
}

\begin{abstract}
Preliminary bulk and clay mineralogy data as determined by XRD as well as accumulation rates of quartz and feldspar at Oki Ridge Site 798 give important information about the paleoclimate in Asia and transport mechanisms of the siliciclastic sediment fraction. The (?uppermost Miocene/) lowermost Pliocene sediments at ODP Site 798 are characterized by high amounts of feldspars, smectites, and additionally abundant sand-sized terrigenous components. This is taken as an indication for turbiditycurrent-influenced deposition and a source area of the material different from that of the upper Pliocene/Pleistocene sediments. A distinct increase in accumulation rates for quartz and feldspar occurs near the Gauss/Matuyama boundary. We attribute this prominent shift to higher eolian sediment supply, caused by the increased aridification of Asia, which was probably triggered by the development of major Northern Hemisphere glaciation near $2.5 \mathrm{Ma}$. The late Pliocene/Pleistocene interval is characterized by short-term "Milankovitch-type" cyclic changes in terrigenous sediment composition most probably reflecting glacial/interglacial arid/humid climate cycles.
\end{abstract}

\section{INTRODUCTION}

During Leg 128 of the Ocean Drilling Program (ODP), three sites were drilled in the Japan Sea with the purpose of reconstructing both the development of the back-arc basin and the origin and genesis of its sediments in relation to the regional paleoclimatic and paleoceanographic evolution (Ingle, Suyehiro, von Breymann, et al., 1990). As shown in Figure 1, the sites were drilled in the Kita-Yamato Trough within the larger Yamato Rise (Site 799), in the northern part of Yamato Basin (Site 794) and - to recover a paleoceanographic reference section - on top of Oki Ridge (Site 798).

The major objective of this study is to reconstruct climate-induced changes in terrigenous sediment supply from the Asian continent in order to create an important link between continental loess records in Asia (e.g., Burbank and Jijun, 1985; Kukla, 1987; Tungsheng, 1988) and eolo-marine records in the Pacific Ocean (e.g., Rea and Janecek, 1982; Pisias and Leinen, 1984; Hovan et al., 1989). The study is based on determinations of accumulation rates and composition of terrigenous matter (bulk and clay mineralogy).

\section{GEOLOGICAL SETTING AND MODERN TERRIGENOUS SEDIMENT SUPPLY AT SITE 798}

Site $798\left(37.04^{\circ} \mathrm{N}, 134.80^{\circ} \mathrm{E} ; 903 \mathrm{~m}\right.$ water depth $)$ is located in the southeastern Japan Sea, about $160 \mathrm{~km}$ north of the western coast of Honshu in a small sediment-filled graben on top of Oki Ridge (Fig. 1). This shallow-water position, well above the modern calcite compensation depth (CCD) (1500-2200 m; Ujiie and Ichikura, 1973) and on a structurally isolated height (Fig. 1), was chosen to obtain a carbonate-rich sequence undiluted by coarse-grained gravity-flow sediments common to the basinal areas (Ingle, Suyehiro, von Breymann, et al., 1990). At Site 798, a 517-m-thick pelagic to hemipelagic sediment sequence of (latest Miocene/) early Pliocene to Holocene age was drilled (Ingle, Suyehiro, von Breymann, et al., 1990; L. Burckle, pers. comm., 1990). Diatomaceous ooze, diatomaceous clay, silty clay, clay, and siliceous claystone are the predominant sediments

\footnotetext{
${ }^{1}$ Pisciotto, K. A., Ingle, J. C., Jr., von Breymann, M. T., Barron, J., et al., 1992. Proc. ODP, Sci. Results, 127/128, Pt. 1: College Station, TX (Ocean Drilling Program).

${ }^{2}$ Alfred-Wegener-Institute for Polar and Marine Research, Columbusstrasse, W-2850 Bremerhaven, Federal Republic of Germany.
}

with common foraminifers and calcareous nannofossils in the upper $220 \mathrm{~m}$. In addition, this upper $220 \mathrm{~m}$ is characterized by rhythmic changes between dark, laminated, diatom- and organic-carbon-rich intervals and light, non-bioturbated to intensely bioturbated, clay-rich intervals (see also Föllmi et al., 1990). The intercalated volcanic ash layers show a maximum occurrence between 0.9 and $0.3 \mathrm{Ma}$ (Ingle, Suyehiro, von Breymann, et al., 1990). Shipboard paleomagnetic and biostratigraphic data (Ingle, Suyehiro, von Breymann, et al., 1990) give sedimentation rates in the range of $9.2-21.7 \mathrm{~cm} / 1000 \mathrm{yr}$ with an average sedimentation rate of $12 \mathrm{~cm} / 1000 \mathrm{yr}$ for the entire sequence of Site 798.

Terrigenous sediment supply to the Japan Sea is strongly influenced by the prevailing westerly winds (Fig. 2; Tungsheng, 1988) as well as the Pacific drainage system of major rivers from the Asian continent and the Japanese Islands. The sources of the transported sediment components at Site 798 can be derived from distant, arid areas, such as the Gobi desert region, as well as more proximal coastal areas surrounding the Sea of Japan (Fig. 2). Because of its isolated position far away from the continent, the modern terrigenous sediment supply at Site 798 is restricted mainly to an eolian input. Thus, the investigation of accumulation rates, composition, and grain size of these terrigenous sediment components may provide information about the paleoclimate of the source area and intensity of the wind system (e.g., Sarnthein et al., 1981, 1982; Stein, 1985a, 1985b; Rea and Janecek, 1982). Furthermore, correlation of the deposition of wind-blown loess in central and eastern China, eolian sediments in the North Pacific Ocean, and the marine delta ${ }^{18} \mathrm{O}$ record (e.g., the global climate record) indicates a link between changes in aridity in Asia and global glacial/interglacial climate cycles (e.g., Kukla et al., 1988; Tungsheng, 1988; Hovan et al., 1989), which should be recorded in the sediments at Site 798.

\section{METHODS \\ Bulk Mineralogy}

After $15 \mathrm{wt} \%$ corundum was added as an internal standard, all samples were ground $15 \mathrm{~min}$ using a grinding mill in order to get well-homogenized samples. X-ray-diffraction (XRD) measurements were performed on randomly oriented, pressed-powder slides by means of a Siemens XRD system (D 501). The diffractometer has copper $\mathrm{K} \alpha$ radiation combined with a $\mathrm{Na}$ Iodide detector. 


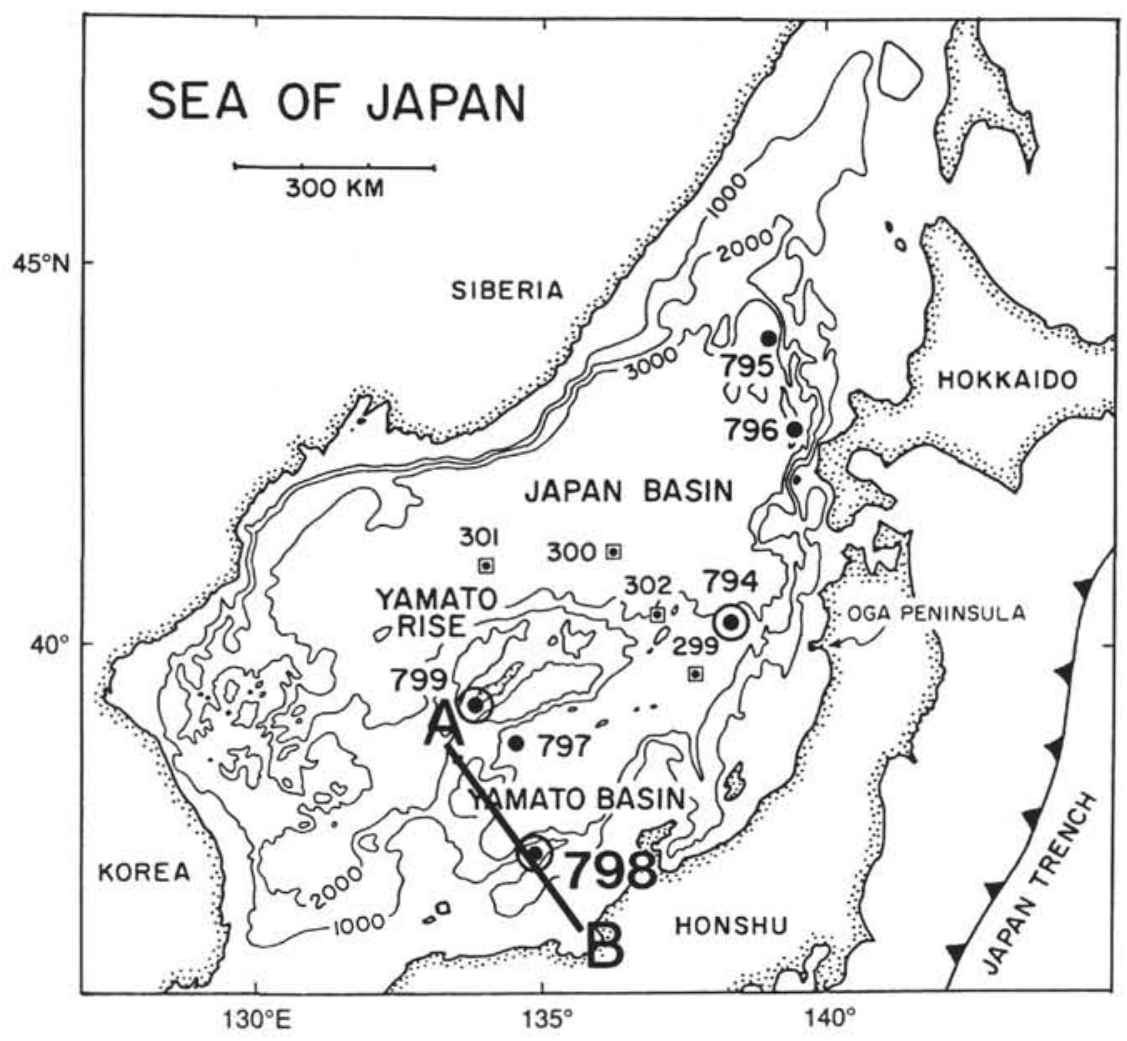

A

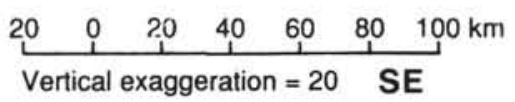

NW

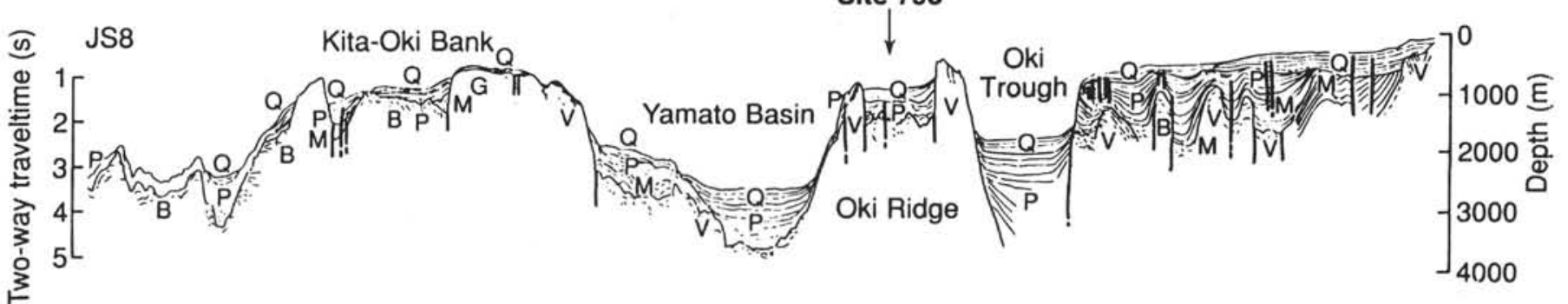

Figure 1. Location map (top) of DSDP Leg 31 Sites 299-302 and ODP Leg 127 Sites 794-797, and Leg 128 Sites 798 and 799, and 794, which were returned to for drilling. Interpreted northwest-southeast single-channel seismic-reflection profile (bottom) showing the location of Hole 798B at the top of Oki Ridge. The rock units are $\mathrm{G}=$ granite, $\mathrm{V}=$ volcanic rock, $\mathrm{B}=$ unknown basement rock, $\mathrm{M}=$ Miocene sediments, $\mathrm{P}=\mathrm{Pliocene}$ sediments, $\mathrm{Q}=\mathrm{Quaternary}$ sediments (profile from Tanaki, 1988).

For the bulk mineralogy, the samples were measured from $2^{\circ}$ to $62^{\circ}$ two theta $(2 \theta)$ at $0.03^{\circ}$ steps/s. Quartz and feldspar data, presented in this study, are given in weight percentages of bulk sediment with a relative error of $\leq 5 \mathrm{wt} \%$ (for the method of quantitative and qualitative determination of bulk mineralogy see Emmermann et al., 1989).

\section{Clay Mineralogy}

After the dissolution of carbonate by $50 \%$ acetic acid, the $<2-\mu \mathrm{m}$ fraction was separated by the Atterberg method (Müller, 1967).
After the addition of $1 \mathrm{wt} \% \mathrm{MoS}_{2}$ as an internal standard, the samples were sucked onto cellulose nitrate filters under vacuum conditions to produce texturally oriented samples. These samples were measured by means of a Phillips PW1700 XRD system with cobalt $\mathrm{K} \alpha$ radiation. The measurements were performed on both untreated samples and glycolated samples from $2^{\circ}$ to $40^{\circ} 2 \theta$ at $0.02^{\circ}$-step width $/ 2 \mathrm{~s}$. Additionally, each sample was measured between $28^{\circ}$ and $30.5^{\circ} 2 \theta$ at $0.005^{\circ}$-step width $/ 2 \mathrm{~s}$ to separate the kaolinite and chlorite more accurately.

Semiquantitative evaluations were based on peak heights, which were multiplied by "Biscaye-factors" (Biscaye, 1965): smectite, 17 


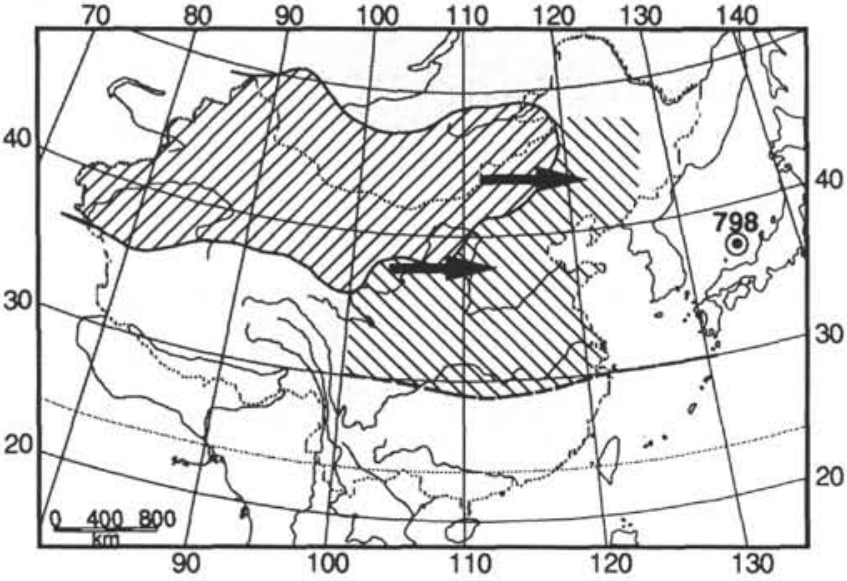

Source Area of Loess

Chinese Border

Deposition Area of Loess

- Site Position

\section{Westerlies}

Figure 2. Source region and deposition region of loess and prevailing westerly winds (after Tungsheng, 1988).

$\AA$ glycolated peak height $(\times 1)$; illite, $10 \AA$ peak height $(\times 4)$, and chlorite-kaolinite, $7 \AA$ peak height $(\times 2)$. The $7 \AA$-peak is separated into a kaolinite and chlorite proportion using the intensity ratio of the kaolinite (002)-peak (3.58 $\AA$ ) and the chlorite (004)-peak ( $3.54 \AA$ ) (for discussion of the method see Gibbs, 1967; Elverhøi and Rønningsland, 1978). The sum of the weighted peak heights accounts for $100 \%$ of the clay mineralogy of the sample. A more detailed study of clay (and bulk) mineralogy based on peak areas is in progress (Dersch, unpubl. data).

\section{Calculation of Accumulation Rates}

Mass-accumulation rates were calculated according to Van Andel et al. (1975), using physical-property and mean sedimentation rate data from Ingle, Suyehiro, von Breymann, et al. (1990) and L. Burckle (pers. comm., 1990), as follows:

$$
\text { MAR = LSR }(\text { WBD-1.026 PO/100), }
$$

where MAR $=$ mass accumulation rate $\left(\mathrm{g} \mathrm{cm}^{-2} \mathrm{k} \cdot \mathrm{y} \cdot{ }^{-1}\right), \mathrm{LSR}=$ linear sedimentation rate $(\mathrm{cm} / 1000 \mathrm{yr}), \mathrm{WBD}=$ wet-bulk density $\left(\mathrm{g} / \mathrm{cm}^{3}\right)$, and $\mathrm{PO}=$ porosity $(\%)$. The MAR of quartz and feldspar can then be calculated by multiplying the MAR by the weight percent value of quartz and feldspar, respectively. Using these accumulation rates, dilution effects by other components can be excluded. Furthermore, the compaction problem can be eliminated and a comparison of sediment accumulation on both a temporal and spatial basis is possible, whereas further diagenetic influence has not been taken into account.

These calculations were performed on the basis of an average sample spacing of $1.50 \mathrm{~m}$, approximately $12,000 \mathrm{yr}$, respectively. In Cores $798-13 \mathrm{H}$ to $798-15 \mathrm{H}$, a time resolution of approximately 3000 yr was reached, using sampling intervals of $0.30 \mathrm{~m}$.

\section{RESULTS}

\section{Composition of the Siliciclastic Sediment Fraction}

In order to get information about the composition of the siliciclastic (i.e., terrigenous) sediment fraction the amounts of quartz and feldspar as well as illite, chlorite, kaolinite, and smectite were determined by XRD (Tables 1 and 2).

The entire sediment sequence at Site 798 is characterized by quartz contents ranging between $5 \%$ and $20 \%$ (Fig. 3). At about 290 m below seafloor (mbsf) (i.e., near the Gauss/Matuyama boundary), a distinct increase in the amplitude of fluctuations occurs. Thus, the upper 290 $\mathrm{m}$ (i.e., the last $2.47 \mathrm{Ma}$ ) show distinct short-term fluctuations. A section within the detailed sampling interval between 115 and 143 mbsf reveals a 40,000-yr cycle (Fig. 3; for details of the short-term "Milankovitch-type" cycles, deduced from the logging data, see deMenocal et al., this volume).

The feldspar content varies between $2 \%$ and $20 \%$ (Fig. 3). Maximum values of $15 \%-20 \%$ are concentrated in the lowermost $80 \mathrm{~m}$ (440-520 mbsf), between 120 and $200 \mathrm{mbsf}$, and in the upper $40 \mathrm{~m}$ of the record.

Most of the quartz/feldspar ratios vary between 0.5 and 3 (Fig. 3). Low ratios are typical in the lowermost $80 \mathrm{~m}$ of the sediment sequence (ratios of $0.5-1.5$ ) and in the uppermost $100 \mathrm{~m}$ (ratios of $0.5-2$ ). The higher ratios of $>2$ are found between 420 and $120 \mathrm{mbsf}$. Isolated peaks with ratios of more than 4 occur at 350,270 , and $150 \mathrm{mbsf}$.

Clay mineral data are shown in Figure 4. In the upper $413 \mathrm{~m}$, the clay fraction at Site 798 is dominated by illite with values between $60 \%$ and $88 \%$ (mean value: $78 \%$ ) whereas the lowermost part shows distinctly lower values between $30 \%$ and $60 \%$ (mean value: $42 \%$; Fig. 4).

The chlorite content varies between about $5 \%$ and $27 \%$ (Fig. 4). A short sequence in the lowermost part of the sediment record with values between $4 \%$ and $14 \%$ ( $500-320 \mathrm{mbsf})$ is followed by a distinct increase in both maximum values and amplitude near $320 \mathrm{mbsf}$ $(0 \%-27 \%)$.

In general, kaolinite content ranges between $0 \%$ and $12 \%$ (Fig. 4). The lowermost part of the section ( $500-325 \mathrm{mbsf})$ is characterized by more constant values between $3 \%$ and $7 \%$, whereas the upper part (325-0 mbsf) displays higher fluctuations with values ranging from $0 \%$ to $12 \%$.

Smectite values range between $0 \%$ and $63 \%$ (Fig. 4). The smectite record can be divided into two sections. The highest values occur in the lowest part (500-423 mbsf) ranging from $23 \%$ to $63 \%$, whereas near 413 mbsf a distinct decrease to values between $0 \%$ and $22 \%$ occurs.

Kaolinite/illite ratios range between 0 and 0.31 , and smectite/illite ratios show expansion between 0 and 2.1. The highest smectite/illite ratios of 0.9-2.1 are typical for the lowermost part of the record (Table 2.).

Based on first results of peak area evaluation, the trends for illite and smectite are similar to those based on peak height, although differences occur in absolute number: illite values vary between $30 \%$ and $70 \%$, whereas smectite values vary between $10 \%$ and $70 \%$. Chlorite and kaolinite values display variations between $5 \%$ and $20 \%$ (Dersch, unpubl. data).

\section{Accumulation Rates of Quartz and Feldspars}

In order to quantify the terrigenous sediment input at Site 798, the accumulation rates of quartz and feldspars have been calculated.

The accumulation rates of quartz vary between about 0.5 and 2.5 $\mathrm{g} \mathrm{cm}^{-2}$ k.y. ${ }^{-1}$ (Fig. 5). Low-amplitude variations between 0.5 and 1.5 $\mathrm{g} \mathrm{cm}^{-2} \mathrm{k.y} .^{-1}$ are typical for the early to early late Pliocene, i.e., 5-2.4 Ma. If the lowermost part of the sediment sequence of Site 798 is of late Miocene age (about 6 Ma; L. Burckle, pers. comm., 1990), accumulation-rate values would decrease to $0.35-1.05 \mathrm{~g} \mathrm{~cm}^{-2} \mathrm{k} . \mathrm{y}^{-1}$. The last $2.4 \mathrm{Ma}$ are characterized by increased amplitude of variations and increased maximum values of quartz-accumulation rates.

The feldspar accumulation rates vary between 0.1 and $2.5 \mathrm{~g} \mathrm{~cm}^{-2}$ k.y. ${ }^{-1}$ (Fig. 5), whereas the lowermost section from 5 to $4.3 \mathrm{Ma}$ displays the highest rates (range: $0.7-2.21 \mathrm{~g} \mathrm{~cm}^{-2} \mathrm{k} \cdot \mathrm{y}^{-1}$; mean value: $\left.1.1 \mathrm{~g} \mathrm{~cm}^{-2} \mathrm{k} . \mathrm{y}^{-1}\right)$. The values would decrease to $0.49-1.55 \mathrm{~g} \mathrm{~cm}^{-2}$ 
Table 1. Bulk accumulation and accumulation rates of quartz, and feldspar, quartz, and feldspar content of the bulk mineralogy, and quartz/feldspar ratios.

\begin{tabular}{|c|c|c|c|c|c|c|c|c|}
\hline $\begin{array}{l}\text { Hole, core, section, } \\
\text { interval }(\mathrm{cm})\end{array}$ & $\begin{array}{l}\text { Depth } \\
\text { (mbsf) }\end{array}$ & $\begin{array}{l}\text { Age } \\
(\mathrm{Ma})\end{array}$ & $\begin{array}{c}\text { Bulk } \\
\text { accumulation }\end{array}$ & Quartz & $\begin{array}{l}\text { Feldspar } \\
\mathrm{g} / \mathrm{cm}^{2} / \mathrm{k} . \mathrm{y} .\end{array}$ & $\begin{array}{l}\text { Quartz } \\
\text { (wt\%) }\end{array}$ & $\begin{array}{l}\text { Feldspar } \\
(w t \%)\end{array}$ & $\begin{array}{l}\text { Quartz/Feldspar } \\
\text { ratio }\end{array}$ \\
\hline $798 \mathrm{~A}-1 \mathrm{H}-1,39-41$ & 0.39 & 0.003 & 8.29 & 1.27 & 1.14 & 15.3 & 13.8 & 1.1 \\
\hline $798 \mathrm{~A}-1 \mathrm{H}-2,46-51$ & 1.96 & 0.016 & 8.09 & 1.21 & 0.72 & 15.0 & 8.9 & 1.7 \\
\hline $798 \mathrm{~A}-1 \mathrm{H}-3,37-39$ & 3.37 & 0.028 & 7.86 & 0.96 & 0.73 & 12.2 & 9.3 & 1.3 \\
\hline $798 \mathrm{~A}-1 \mathrm{H}-4,38-45$ & 4.88 & 0.041 & 8.29 & 1.09 & 0.91 & 13.1 & 11.0 & 1.2 \\
\hline $798 \mathrm{~A}-1 \mathrm{H}-5,38-40$ & 6.38 & 0.053 & 7.95 & 0.88 & 1.38 & 11.1 & 17.3 & 0.6 \\
\hline $798 \mathrm{~A}-1 \mathrm{H}-6,38-44$ & 7.88 & 0.066 & 7.62 & 0.80 & 0.70 & 10.5 & 9.2 & 1.1 \\
\hline $798 \mathrm{~A}-2 \mathrm{H}-2,46-51$ & 11.26 & 0.094 & 8.88 & 1.12 & 0.77 & 12.6 & 8.7 & 1.5 \\
\hline $798 \mathrm{~A}-2 \mathrm{H}-3,46-49$ & 12.76 & 0.106 & 9.91 & 1.63 & 1.09 & 16.4 & 11.0 & 1.5 \\
\hline $798 \mathrm{~A}-2 \mathrm{H}-4,4-9$ & 13.84 & 0.115 & 9.73 & 1.42 & 0.77 & 14.6 & 7.9 & 1.9 \\
\hline $798 \mathrm{~A}-3 \mathrm{H}-1,71-73$ & 19.01 & 0.158 & 6.28 & 0.73 & 0.70 & 11.6 & 11.1 & 1.1 \\
\hline $798 \mathrm{~A}-3 \mathrm{H}-2,49-51$ & 20.29 & 0.169 & 9.50 & 1.45 & 1.80 & 15.3 & 18.9 & 0.8 \\
\hline $798 \mathrm{~A}-3 \mathrm{H}-3,49-51$ & 21.79 & 0.182 & 8.47 & 1.09 & 0.73 & 12.9 & 8.6 & 1.5 \\
\hline $798 \mathrm{~A}-3 \mathrm{H}-4,49-51$ & 23.29 & 0.194 & 9.74 & 1.76 & 1.46 & 18.1 & 15.0 & 1.2 \\
\hline $798 \mathrm{~A}-3 \mathrm{H}-5,53-55$ & 24.83 & 0.207 & 9.45 & 1.24 & 1.17 & 13.1 & 12.4 & 1.1 \\
\hline $798 \mathrm{~A}-3 \mathrm{H}-6,49-51$ & 26.29 & 0.219 & 9.40 & 1.54 & 1.42 & 16.4 & 15.1 & 1.1 \\
\hline $798 \mathrm{~A}-4 \mathrm{H}-2,29-31$ & 29.09 & 0.242 & 10.59 & 1.44 & 1.45 & 13.6 & 13.7 & 1.0 \\
\hline $798 \mathrm{~A}-4 \mathrm{H}-3,29-31$ & 30.59 & 0.255 & 10.22 & 1.70 & 0.81 & 16.6 & 7.9 & 2.1 \\
\hline $798 \mathrm{~A}-4 \mathrm{H}-4,59-63$ & 32.39 & 0.270 & 10.10 & 1.15 & 0.66 & 11.4 & 6.5 & 1.8 \\
\hline $798 \mathrm{~A}-5 \mathrm{H}-2,80-82$ & 38.83 & 0.324 & 10.53 & 1.02 & 1.50 & 9.7 & 14.2 & 0.7 \\
\hline $798 \mathrm{~A}-5 \mathrm{H}-3,49-5 \mathrm{I}$ & 39.93 & 0.333 & 8.92 & 0.88 & 0.44 & 9.9 & 4.9 & 2.0 \\
\hline $798 \mathrm{~A}-5 \mathrm{H}-4,86-88$ & 41.66 & 0.347 & 9.23 & 1.02 & 0.60 & 11.0 & 6.5 & 1.7 \\
\hline $798 \mathrm{~A}-5 \mathrm{H}-6.33-35$ & 43.94 & 0.365 & 10.51 & 1.38 & 1.16 & 13.1 & 11.0 & 1.2 \\
\hline $798 \mathrm{~A}-5 \mathrm{H}-7,7-11$ & 45.09 & 0.374 & 9.85 & 0.85 & 0.64 & 8.6 & 6.5 & 1.3 \\
\hline $798 \mathrm{~A}-6 \mathrm{H}-2,49-54$ & 48.10 & 0.398 & 10.54 & 1.01 & 0.83 & 9.6 & 7.9 & 1.2 \\
\hline $798 \mathrm{~A}-6 \mathrm{H}-3,7-9$ & 49.12 & 0.407 & 10.50 & 1.07 & 0.95 & 10.2 & 9.0 & 1.1 \\
\hline $798 \mathrm{~A}-6 \mathrm{H}-3,18-20$ & 49.23 & 0.407 & 10.50 & 1.18 & 0.72 & 11.2 & 6.9 & 1.6 \\
\hline $798 \mathrm{~A}-6 \mathrm{H}-3,28-30$ & 49.32 & 0.408 & 10.50 & 1.08 & 0.79 & 10.3 & 7.5 & 1.4 \\
\hline $798 \mathrm{~A}-6 \mathrm{H}-3,35-37$ & 49.39 & 0.409 & 10.50 & 1.00 & 0.79 & 9.5 & 7.5 & 1.3 \\
\hline $798 \mathrm{~A}-6 \mathrm{H}-4,4-9$ & 50.52 & 0.418 & 9.74 & 1.02 & 0.68 & 10.5 & 7.0 & 1.5 \\
\hline $798 \mathrm{~A}-6 \mathrm{H}-6,84-89$ & 54.14 & 0.447 & 10.50 & 1.81 & 1.19 & 17.2 & 11.3 & 1.5 \\
\hline $798 \mathrm{~A}-7 \mathrm{H}-2,130-135$ & 58.06 & 0.485 & 6.71 & 0.83 & 0.79 & 12.3 & 11.8 & 1.0 \\
\hline $798 \mathrm{~A}-7 \mathrm{H}-3,51-53$ & 58.66 & 0.492 & 6.43 & 0.62 & 0.30 & 9.7 & 4.6 & 2.1 \\
\hline $798 \mathrm{~A}-7 \mathrm{H}-4,50-55$ & 59.92 & 0.505 & 8.39 & 1.21 & 0.73 & 14.4 & 8.7 & 1.7 \\
\hline $798 \mathrm{~A}-7 \mathrm{H}-6,65-70$ & 62.57 & 0.535 & 7.42 & 0.75 & 0.71 & 10.1 & 9.6 & 1.1 \\
\hline $798 \mathrm{~A}-7 \mathrm{H}-7,66-68$ & 63.85 & 0.549 & 7.18 & 0.75 & 0.44 & 10.4 & 6.1 & 1.7 \\
\hline $798 \mathrm{~A}-7 \mathrm{H}-8,55-60$ & 65.02 & 0.561 & 7.50 & 1.00 & 0.85 & 13.3 & 11.3 & 1.2 \\
\hline $798 \mathrm{~A}-8 \mathrm{H}-3,32-34$ & 68.29 & 0.598 & 7.25 & 0.53 & 0.00 & 7.3 & 0.0 & \\
\hline $798 \mathrm{~A}-8 \mathrm{H}-5,61-63$ & 71.26 & 0.630 & 7.89 & 0.96 & 0.84 & 12.2 & 10.6 & 1.2 \\
\hline $798 \mathrm{~A}-8 \mathrm{H}-7,64-66$ & 73.99 & 0.660 & 8.16 & 0.71 & 0.69 & 8.7 & 8.4 & 1.0 \\
\hline $798 \mathrm{~A}-8 \mathrm{H}-8,45-47$ & 75.17 & 0.673 & 7.26 & 0.69 & 0.58 & 9.5 & 8.0 & 1.2 \\
\hline $798 \mathrm{~A}-9 \mathrm{H}-1,54-56$ & 75.30 & 0.675 & 8.96 & 0.88 & 0.52 & 9.8 & 5.8 & 1.7 \\
\hline $798 \mathrm{~A}-9 \mathrm{H}-2,29-31$ & 76.46 & 0.687 & 8.32 & 0.94 & 0.49 & 11.3 & 5.9 & 1.9 \\
\hline $798 \mathrm{~A}-9 \mathrm{H}-3,55-57$ & 78.09 & 0.705 & 6.64 & 0.90 & 0.44 & 13.5 & 6.6 & 2.1 \\
\hline $798 \mathrm{~A}-9 \mathrm{H}-4.54-56$ & 79.47 & 0.720 & 6.55 & 0.54 & 0.52 & 8.2 & 7.9 & 1.0 \\
\hline $798 \mathrm{~A}-9 \mathrm{H}-5,54-56$ & 80.86 & 0.741 & 3.68 & 0.35 & 0.18 & 9.4 & 4.9 & 1.9 \\
\hline $798 \mathrm{~A}-9 \mathrm{H}-6,53-58$ & 82.24 & 0.771 & 3.51 & 0.32 & 0.22 & 9.2 & 6.4 & 1.4 \\
\hline $798 \mathrm{~A}-10 \mathrm{H}-2,0-5$ & 85.74 & 0.839 & 4.30 & 0.40 & 0.34 & 9.4 & 8.0 & 1.2 \\
\hline $798 \mathrm{~A}-10 \mathrm{H}-3,40-45$ & 87.44 & 0.866 & 4.40 & 0.40 & 0.36 & 9.2 & 8.1 & 1.1 \\
\hline $798 \mathrm{~A}-10 \mathrm{H}-5,103-108$ & 90.69 & 0.913 & 9.28 & 0.93 & 0.90 & 10.0 & 9.7 & 1.0 \\
\hline $798 \mathrm{~A}-10 \mathrm{H}-7,20-25$ & 92.63 & 0.927 & 10.00 & 1.00 & 0.48 & 10.0 & 4.8 & 2.1 \\
\hline $798 \mathrm{~A}-10 \mathrm{H}-7,128-133$ & 93.59 & 0.935 & 11.69 & 1.10 & 0.91 & 9.4 & 7.8 & 1.2 \\
\hline $798 \mathrm{~A}-10 \mathrm{H}-8,63-65$ & 94.36 & 0.940 & 11.94 & 1.21 & 0.57 & 10.1 & 4.8 & 2.1 \\
\hline $798 \mathrm{~A}-11 \mathrm{H}-1,97-99$ & 95.02 & 0.945 & 9.00 & 0.77 & 0.83 & 8.6 & 9.2 & 0.9 \\
\hline $798 \mathrm{~A}-11 \mathrm{H}-2,0-5$ & 95.53 & 0.949 & 8.00 & 0.80 & 0.41 & 10.0 & 5.1 & 2.0 \\
\hline $798 \mathrm{~A}-11 \mathrm{H}-2,80-84$ & 96.29 & 0.954 & 9.82 & 1.11 & 1.10 & 11.3 & 11.2 & 1.0 \\
\hline $798 \mathrm{~A}-11 \mathrm{H}-4,2-4$ & 98.40 & 0.970 & 10.50 & 1.00 & 0.84 & 9.5 & 8.0 & 1.2 \\
\hline $798 \mathrm{~A}-11 \mathrm{H}-4,86-90$ & 99.20 & 0.976 & 9.67 & 0.99 & 0.71 & 10.2 & 7.3 & 1.4 \\
\hline $798 \mathrm{~A}-11 \mathrm{H}-6,102-107$ & 102.21 & 0.997 & 13.44 & 1.79 & 0.98 & 13.3 & 7.3 & 1.8 \\
\hline $798 \mathrm{~A}-11 \mathrm{H}-7,72-74$ & 103.35 & 1005 & 11.00 & 0.69 & 0.40 & 6.3 & 3.6 & 1.8 \\
\hline $798 \mathrm{~A}-12 \mathrm{H}-2,30-35$ & 105.35 & 1020 & 10.41 & 0.90 & 0.84 & 8.6 & 8.1 & 1.1 \\
\hline $798 \mathrm{~A}-12 \mathrm{H}-5,0-5$ & 108.97 & 1046 & 9.80 & 0.93 & 0.89 & 9.5 & 9.1 & 1.0 \\
\hline $798 \mathrm{~A}-12 \mathrm{H}-8,52-54$ & 113.30 & 1077 & 11.00 & 1.57 & 0.76 & 14.3 & 6.9 & 2.1 \\
\hline $798 \mathrm{~B}-13 \mathrm{H}-2,61-100$ & 115.31 & 1091 & 14.00 & 2.10 & 1.33 & 15.0 & 9.5 & 1.6 \\
\hline $798 \mathrm{~A}-13 \mathrm{H}-2,98-103$ & 115.78 & 1095 & 15.53 & 2.98 & 2.42 & 19.2 & 15.6 & 1.2 \\
\hline $798 \mathrm{~B}-13 \mathrm{H}-3,0-30$ & 116.07 & 1097 & 14.00 & 1.57 & 0.63 & 11.2 & 4.5 & 2.5 \\
\hline $798 \mathrm{~B}-13 \mathrm{H}-3,90-120$ & 116.84 & 1102 & 12.50 & 1.23 & 0.56 & 9.8 & 4.5 & 2.2 \\
\hline $798 \mathrm{~B}-13 \mathrm{H}-4,30-60$ & 117.61 & 1108 & 12.00 & 1.55 & 0.82 & 12.9 & 6.8 & 1.9 \\
\hline $798 \mathrm{~B}-13 \mathrm{H}-4,90-120$ & 118.12 & 1111 & 11.50 & 1.37 & 0.92 & 11.9 & 8.0 & 1.5 \\
\hline $798 \mathrm{~A}-13 \mathrm{H}-4,74-79$ & 118.42 & 1114 & 11.40 & 1.22 & 0.63 & 10.7 & 5.5 & 2.0 \\
\hline $798 \mathrm{~B}-13 \mathrm{H}-5,90-120$ & 119.41 & 1121 & 10.50 & 1.56 & 0.67 & 14.9 & 6,4 & 2.3 \\
\hline $798 \mathrm{~B}-14 \mathrm{H}-1,87-89$ & 123.94 & 1153 & 10.89 & 1.36 & 1.06 & 12.5 & 9.7 & 1.3 \\
\hline
\end{tabular}


Table 1 (continued).

\begin{tabular}{|c|c|c|c|c|c|c|c|c|}
\hline $\begin{array}{l}\text { Hole, core, section, } \\
\text { interval }(\mathrm{cm})\end{array}$ & $\begin{array}{l}\text { Depth } \\
\text { (mbsf) }\end{array}$ & $\begin{array}{l}\text { Age } \\
(\mathrm{Ma})\end{array}$ & $\begin{array}{c}\text { Bulk } \\
\text { accumulation }\end{array}$ & Quartz & $\begin{array}{l}\text { Feldspar } \\
\mathrm{g} / \mathrm{cm}^{2} / \mathrm{k} . \mathrm{y} .\end{array}$ & $\begin{array}{l}\text { Quartz } \\
\text { (wt\%) }\end{array}$ & $\begin{array}{l}\text { Feldspar } \\
(w t \%)\end{array}$ & $\begin{array}{l}\text { Quartz/Feldspar } \\
\text { ratio }\end{array}$ \\
\hline $798 \mathrm{~B}-14 \mathrm{H}-2,0-40$ & 124.48 & 1157 & 10.80 & 1.25 & 0.67 & 11.6 & 6.2 & 1.9 \\
\hline $798 \mathrm{~B}-14 \mathrm{H}-2,0-40$ & 124.48 & 1157 & 10.80 & 1.24 & 0.79 & 11.5 & 7.3 & 1.6 \\
\hline $798 \mathrm{~A}-14 \mathrm{H}-2,30-34$ & 124.73 & 1159 & 10.82 & 1.07 & 0.91 & 9.9 & 8.4 & 1.2 \\
\hline $798 \mathrm{~B}-14 \mathrm{H}-2,90-120$ & 125.24 & 1162 & 12.50 & 1.31 & 1.45 & 10.5 & 11.6 & 0.9 \\
\hline $798 \mathrm{~B}-14 \mathrm{H}-2,116-120$ & 125.46 & 1164 & 13.10 & 1.44 & 0.69 & 11.0 & 5.3 & 2.1 \\
\hline $798 \mathrm{~B}-14 \mathrm{H}-2,120-150$ & 125.50 & 1164 & 13.00 & 1.55 & 0.77 & 11.9 & 5.9 & 2.0 \\
\hline $798 \mathrm{~B}-14 \mathrm{H}-3,0-30$ & 125.75 & 1166 & 12.50 & 1.64 & 1.25 & 13.1 & 10.0 & 1.3 \\
\hline $798 \mathrm{~B}-14 \mathrm{H}-3,75-108$ & 126.39 & 1171 & 11.00 & 1.43 & 0.72 & 13.0 & 6.5 & 2.0 \\
\hline $798 \mathrm{~B}-14 \mathrm{H}-3,89-91$ & 126.51 & 1172 & 12.94 & 1.82 & 1.76 & 14.1 & 13.6 & 1.0 \\
\hline $798 \mathrm{~B}-14 \mathrm{H}-3,116-150$ & 126.74 & 1173 & 13.00 & 1.44 & 1.08 & 11.1 & 8.3 & 1.3 \\
\hline $798 \mathrm{~B}-14 \mathrm{H}-4,39-41$ & 127.36 & 1178 & 13.22 & 1.47 & 1.53 & 11.1 & 11.6 & 1.0 \\
\hline 798B-14H-4, 60-90 & 127.54 & 1179 & 12.50 & 1.03 & 0.68 & 8.2 & 5.4 & 1.5 \\
\hline $798 \mathrm{~A}-14 \mathrm{H}-4,62-66$ & 127.73 & 1180 & 12.26 & 1.37 & 0.85 & 11.2 & 6.9 & 1.6 \\
\hline $798 \mathrm{~B}-14 \mathrm{H}-4,90-120$ & 127.79 & 1181 & 12.20 & 1.02 & 0.60 & 8.4 & 4.9 & 1.7 \\
\hline $798 \mathrm{~B}-14 \mathrm{H}-4,120-150$ & 128.05 & 1183 & 12.50 & 1.24 & 0.91 & 9.9 & 7.3 & 1.4 \\
\hline $798 \mathrm{~B}-14 \mathrm{H}-4,120-150$ & 128.05 & 1183 & 12.50 & 1.26 & 0.98 & 10.1 & 7.8 & 1.3 \\
\hline $798 \mathrm{~B}-14 \mathrm{H}-5,0-30$ & 128.30 & 1184 & 13.00 & 1.34 & 0.70 & 10.3 & 5.4 & 1.9 \\
\hline $798 \mathrm{~B}-14 \mathrm{H}-5,30-60$ & 128.56 & 1186 & 13.00 & 1.05 & 0.83 & 8.1 & 6.4 & 1.3 \\
\hline $798 \mathrm{~A}-14 \mathrm{H}-6,49-53$ & 130.32 & 1199 & 12.80 & 1.36 & 0.56 & 10.6 & 4.4 & 2.4 \\
\hline $798 \mathrm{~B}-14 \mathrm{H}-6,90-120$ & 130.34 & 1199 & 12.80 & 1.37 & 1.28 & 10.7 & 10.0 & 1.1 \\
\hline $798 \mathrm{~B}-14 \mathrm{H}-6,120-150$ & 130.60 & 1201 & 12.80 & 1.60 & 1.05 & 12.5 & 8.2 & 1.5 \\
\hline $798 \mathrm{~B}-14 \mathrm{H}-7,65-67$ & 131.41 & 1207 & 13.22 & 1.59 & 1.16 & 12.0 & 8.8 & 1.4 \\
\hline $798 \mathrm{~B}-14 \mathrm{H}-7,137-150$ & 132.02 & 1211 & 13.50 & 1.66 & 1.46 & 12.3 & 10.8 & 1.1 \\
\hline $798 \mathrm{~B}-14 \mathrm{H}-8,0-30$ & 132.13 & 1212 & 13.50 & 1.77 & 1.07 & 13.1 & 7.9 & 1.7 \\
\hline $798 \mathrm{~B}-14 \mathrm{H}-8,30-60$ & 132.38 & 1214 & 13.20 & 1.69 & 0.92 & 12.8 & 7.0 & 1.8 \\
\hline $798 \mathrm{~B}-14 \mathrm{H}-8,65-67$ & 132.68 & 1216 & 13.19 & 1.82 & 1.16 & 13.8 & 8.8 & 1.6 \\
\hline $798 \mathrm{~A}-14 \mathrm{H}-8,22-26$ & 132.79 & 1217 & 9.78 & 1.09 & 0.82 & 11.1 & 8.4 & 1.3 \\
\hline $798 \mathrm{~B}-14 \mathrm{H}-8,110-134$ & 133.06 & 1219 & 11.94 & 0.80 & 2.23 & 6.7 & 18.7 & 0.4 \\
\hline $798 \mathrm{~B}-15 \mathrm{H}-2,0-30$ & 134.17 & 1226 & 10.00 & 1.18 & 0.84 & 11.8 & 8.4 & 1.4 \\
\hline $798 \mathrm{~A}-15 \mathrm{H}-2,35-39$ & 134.34 & 1228 & 9.56 & 1.02 & 0.36 & 10.7 & 3.8 & 2.8 \\
\hline $798 \mathrm{~B}-15 \mathrm{H}-2,30-80$ & 134,42 & 1228 & 10.00 & 1.35 & 0.50 & 13.5 & 5.0 & 2.7 \\
\hline $798 \mathrm{~B}-15 \mathrm{H}-2,80-120$ & 134.84 & 1231 & 10.50 & 1.53 & 0.72 & 14.6 & 6.9 & 2.1 \\
\hline 798B-15H-2, 109-111 & 135.09 & 1233 & 11.33 & 1.68 & 0.88 & 14.8 & 7.8 & 1.9 \\
\hline $798 \mathrm{~B}-15 \mathrm{H}-3,22-24$ & 135.62 & 1237 & 11.70 & 1.30 & 0.84 & 11.1 & 7.2 & 1.5 \\
\hline $798 \mathrm{~A}-15 \mathrm{H}-4,30-34$ & 136.79 & 1245 & 9.62 & 0.80 & 0.30 & 8.3 & 3.1 & 2.7 \\
\hline $798 \mathrm{~B}-15 \mathrm{H}-4,74-76$ & 137.33 & 1249 & 10.50 & 1.55 & 0.97 & 14.8 & 9.2 & 1.6 \\
\hline 798B-15H-5, 60-90 & 138.48 & 1257 & 10.50 & 0.92 & 0.36 & 8.8 & 3.4 & 2.6 \\
\hline 798B-15H-5, 74-76 & 138.60 & 1258 & 9.64 & 0.94 & 0.56 & 9.8 & 5.8 & 1.7 \\
\hline $798 \mathrm{~A}-15 \mathrm{H}-6,15-19$ & 139.16 & 1262 & 12.16 & 1.91 & 1.41 & 15.7 & 11.6 & 1.4 \\
\hline $798 \mathrm{~B}-15 \mathrm{H}-6,0-30$ & 139.24 & 1263 & 12.00 & 1.25 & 0.56 & 10.4 & 4.7 & 2.2 \\
\hline 798B-15H-6, 74-76 & 139.87 & 1267 & 11.02 & 1.05 & 0.00 & 9.5 & 0.0 & \\
\hline 798B-15H-7, 30-53 & 140.76 & 1274 & 8.50 & 0.75 & 0.34 & 8.8 & 4.0 & 2.2 \\
\hline $798 \mathrm{~B}-15 \mathrm{H}-7,90-120$ & 141.27 & 1277 & 8.50 & 1.33 & 0.51 & 15.7 & 6.0 & 2.6 \\
\hline $798 \mathrm{~B}-15 \mathrm{H}-7,120-150$ & 141.52 & 1279 & 8.50 & 1.29 & 0.48 & 15.2 & 5.6 & 2.7 \\
\hline $798 \mathrm{~A}-15 \mathrm{H}-8,29-33$ & 141.77 & 1281 & 8.50 & 0.78 & 0.22 & 9.2 & 2.6 & 3.5 \\
\hline 798B-15H-8, 75-80 & 142.41 & 1286 & 11.89 & 1.65 & 1.08 & 13.9 & 9.1 & 1.5 \\
\hline $798 \mathrm{~B}-15 \mathrm{H}-8,110-134$ & 142.71 & 1288 & 11.00 & 1.67 & 0.95 & 15.2 & 8.6 & 1.8 \\
\hline $798 B-16 X-1,45-47$ & 143.05 & 1290 & 10.40 & 1.08 & 0.43 & 10.4 & 4.1 & 2.5 \\
\hline $798 B-16 X-2,45-50$ & 144.53 & 1301 & 8.72 & 0.78 & 0.35 & 8.9 & 4.0 & 2.2 \\
\hline $798 \mathrm{~B}-16 \mathrm{X}-4,48-53$ & 147.53 & 1322 & 8.46 & 0.63 & 0.26 & 7.5 & 3.1 & 2.4 \\
\hline 798B-16X-6, 34-39 & 150.36 & 1343 & 7.59 & 0.60 & 0.00 & 7.9 & 0.0 & \\
\hline $798 \mathrm{~B}-16 \mathrm{X}-7,47-49$ & 151.98 & 1354 & 8.00 & 0.65 & 0.16 & 8.1 & 2.0 & 4.1 \\
\hline $798 B-17 X-2,48-54$ & 154.18 & 1370 & 7,40 & 0.44 & 0.27 & 5.9 & 3.7 & 1.6 \\
\hline $798 \mathrm{~B}-17 \mathrm{X}-3,49-51$ & 155.61 & 1380 & 7.13 & 0.45 & 0.00 & 6.3 & 0.0 & \\
\hline $798 \mathrm{~B}-17 \mathrm{X}-4,28-35$ & 156.83 & 1389 & 7.68 & 0.59 & 0.41 & 7.7 & 5.4 & 1.4 \\
\hline $798 \mathrm{~B}-17 \mathrm{X}-5,29-31$ & 158.26 & 1399 & 7.89 & 0.59 & 0.31 & 7.5 & 3.9 & 1.9 \\
\hline $798 B-17 X-6,39-44$ & 159.78 & 1410 & 8.15 & 0.64 & 0.50 & 7.8 & 6.1 & 1.3 \\
\hline $798 \mathrm{~B}-18 \mathrm{X}-1,30-32$ & 162.20 & 1427 & 11.84 & 1.70 & 1.30 & 14.4 & 11.0 & 1.3 \\
\hline $798 \mathrm{~B}-18 \mathrm{X}-3,43-45$ & 165.33 & 1450 & 10.04 & 1.04 & 0.95 & 10.4 & 9.5 & 1.1 \\
\hline $798 \mathrm{~B}-18 \mathrm{X}-5,130-132$ & 169.20 & 1478 & 7.65 & 0.45 & 0.24 & 5.9 & 3.2 & 1.8 \\
\hline $798 \mathrm{~B}-18 \mathrm{X}-6,25-30$ & 169.65 & 1481 & 8.00 & 0.77 & 0.50 & 9.6 & 6.2 & 1.6 \\
\hline $798 B-19 X-1,59-61$ & 172.19 & 1499 & 10.63 & 0.96 & 0.51 & 9.0 & 4.8 & 1.9 \\
\hline $798 \mathrm{~B}-19 \mathrm{X}-2,60-65$ & 173.70 & 1510 & 6.33 & 0.45 & 0.41 & 7.1 & 6.4 & 1.1 \\
\hline 798B-18X-9, 30-35 & 174.20 & 1514 & 8.00 & 0.45 & 0.34 & 5.6 & 4.2 & 1.3 \\
\hline 798B-19X-3, 59-61 & 175.19 & 1521 & 11.78 & 0.86 & 2.00 & 7.3 & 17.0 & 0.4 \\
\hline 798B-19X-5, 51-53 & 178.11 & 1542 & 12.50 & 0.95 & 0.95 & 7.6 & 7.6 & 1.0 \\
\hline 798B-19X-6, 60-65 & 179.70 & 1553 & 13.78 & 1.98 & 1.13 & 14.4 & 8.2 & 1.8 \\
\hline $798 \mathrm{~B}-20 \mathrm{X}-1,39-41$ & 181.62 & 1567 & 10.68 & 1.74 & 0.96 & 16.3 & 9.0 & 1.8 \\
\hline $798 \mathrm{~B}-20 \mathrm{X}-2,40-45$ & 182.86 & 1576 & 12.86 & 2.07 & 1.48 & 16.1 & 11.5 & 1.4 \\
\hline 798B-20X-4, 103-108 & 185.85 & 1597 & 8.84 & 0.69 & 0.49 & 7.8 & 5.5 & 1.4 \\
\hline $798 \mathrm{~B}-20 \mathrm{X}-5,46-48$ & 186.62 & 1603 & 8.74 & 0.98 & 0.48 & 11.2 & 5.5 & 2.0 \\
\hline $798 \mathrm{~B}-20 \mathrm{X}-6,60-65$ & 187.97 & 1612 & 11.00 & 0.36 & 3.04 & 3.3 & 27.6 & 0.1 \\
\hline
\end{tabular}


Table 1 (continued).

\begin{tabular}{|c|c|c|c|c|c|c|c|c|}
\hline $\begin{array}{l}\text { Hole, core, section, } \\
\text { interval }(\mathrm{cm})\end{array}$ & $\begin{array}{l}\text { Depth } \\
\text { (mbsf) }\end{array}$ & $\begin{array}{l}\text { Age } \\
(\mathrm{Ma})\end{array}$ & $\begin{array}{c}\text { Bulk } \\
\text { accumulation }\end{array}$ & Quartz & $\begin{array}{l}\text { Feldspar } \\
\mathrm{g} / \mathrm{cm}^{2} / \mathrm{k} . \mathrm{y} .\end{array}$ & $\begin{array}{l}\text { Quartz } \\
\text { (wt\%) }\end{array}$ & $\begin{array}{l}\text { Feldspar } \\
(w t \%)\end{array}$ & $\begin{array}{c}\text { Quartz/Feldspar } \\
\text { ratio }\end{array}$ \\
\hline $798 \mathrm{~B}-21 \mathrm{X}-2,77-79$ & 193.27 & 1650 & 10.25 & 1.11 & 0.52 & 10.8 & 5.1 & 2.1 \\
\hline $798 \mathrm{~B}-21 \mathrm{X}-3,59-61$ & 194.59 & 1660 & 10.88 & 1.13 & 0.60 & 10.4 & 5.5 & 1.9 \\
\hline $798 \mathrm{~B}-21 \mathrm{X}-4,39-44$ & 195.89 & 1674 & 6.73 & 0.41 & 1.18 & 6.1 & 17.6 & 0.4 \\
\hline $798 \mathrm{~B}-21 \mathrm{X}-6,59-64$ & 199.09 & 1709 & 9.03 & 1.45 & 1.04 & 16.1 & 11.5 & 1.4 \\
\hline 798B-21X-7, 77-79 & 200.77 & 1727 & 5.65 & 0.42 & 0.41 & 7.4 & 7.3 & 1.0 \\
\hline $798 \mathrm{~B}-21 \mathrm{X}-8,19-24$ & 201.69 & 1737 & 6.00 & 0.42 & 0.23 & 7.0 & 3.9 & 1.8 \\
\hline $798 \mathrm{~B}-22 \mathrm{X}-2,30-35$ & 202.07 & 1741 & 6.52 & 0.55 & 0.22 & 8.4 & 3.4 & 2.5 \\
\hline $798 \mathrm{~B}-22 \mathrm{X}-4,17-22$ & 204.41 & 1766 & 5.59 & 0.54 & 0.00 & 9.7 & 0.0 & \\
\hline 798B-22X-6, 90-92 & 207.46 & 1799 & 7.32 & 1.01 & 0.65 & 13.8 & 8.9 & 1.6 \\
\hline $798 \mathrm{~B}-22 X-7,42-47$ & 208.29 & 1808 & 7.02 & 0.86 & 0.86 & 12.3 & 12.3 & 1.0 \\
\hline 798B-22X-8, 77-79 & 209.80 & 1825 & 8.50 & 1.30 & 0.65 & 15.3 & 7.6 & 2.0 \\
\hline $798 \mathrm{~B}-23 \mathrm{X}-2,30-32$ & 211.81 & 1847 & 5.80 & 0.49 & 0.26 & 8.4 & 4.5 & 1.9 \\
\hline $798 B-23 X-5,120-125$ & 216.35 & 1890 & 9.30 & 0.99 & 0.53 & 10.6 & 5.7 & 1.9 \\
\hline $798 \mathrm{~B}-23 \mathrm{X}-6,61-63$ & 217.11 & 1896 & 11.15 & 1.48 & 0.88 & 13.3 & 7.9 & 1.7 \\
\hline $798 \mathrm{~B}-23 \mathrm{X}-8,34-36$ & 219.40 & 1912 & 10.21 & 1.33 & 0.59 & 13.0 & 5.8 & 2.2 \\
\hline 798B-24X-2, 49-54 & 221.95 & 1930 & 9.51 & 1.00 & 0.50 & 10.5 & 5.3 & 2.0 \\
\hline $798 \mathrm{~B}-24 \mathrm{X}-4,49-54$ & 224.89 & 1951 & 9.17 & 0.75 & 0.84 & 8.2 & 9.2 & 0.9 \\
\hline $798 \mathrm{~B}-24 \mathrm{X}-5,50-52$ & 226.37 & 1961 & 9.05 & 0.78 & 0.75 & 8.6 & 8.3 & 1.0 \\
\hline 798B-24X-6, 43-48 & 227.77 & 1971 & 9.84 & 0.90 & 0.84 & 9.1 & 8.5 & 1.1 \\
\hline $798 \mathrm{~B}-24 \mathrm{X}-7,22-24$ & 229.04 & 1980 & 10.38 & 1.14 & 0.56 & 11.0 & 5.4 & 2.0 \\
\hline $798 \mathrm{~B}-25 \mathrm{X}-2,68-73$ & 231.88 & 2000 & 9.25 & 0.80 & 0.31 & 8.7 & 3.4 & 2.6 \\
\hline $798 \mathrm{~B}-25 \mathrm{X}-3,68-70$ & 233.38 & 2010 & 9.69 & 1.07 & 1.04 & 11.0 & 10.7 & 1.0 \\
\hline $798 \mathrm{~B}-25 \mathrm{X}-4,63-67$ & 234.83 & 2021 & 11.32 & 1.42 & 0.85 & 12.5 & 7.5 & 1.7 \\
\hline $798 \mathrm{~B}-27 \mathrm{X}-1,66-68$ & 249.65 & 2125 & 14.13 & 2.44 & 1.61 & 17.3 & 11.4 & 1.5 \\
\hline $798 \mathrm{~B}-27 \mathrm{X}-2,38-43$ & 250.86 & 2134 & 13.65 & 2.10 & 1.87 & 15.4 & 13.7 & 1.1 \\
\hline $798 \mathrm{~B}-27 \mathrm{X}-4,15-20$ & 253.60 & 2153 & 8.89 & 0.90 & 0.53 & 10.1 & 6.0 & 1.7 \\
\hline $798 \mathrm{~B}-27 \mathrm{X}-5,97-99$ & 255.90 & 2169 & 11.43 & 1.05 & 1.23 & 9.2 & 10.8 & 0.9 \\
\hline $798 \mathrm{~B}-27 \mathrm{X}-6,95-100$ & 257.37 & 2179 & 13.37 & 2.02 & 1.06 & 15.1 & 7.9 & 1.9 \\
\hline $798 \mathrm{~B}-27 \mathrm{X}-7,30-32$ & 258.21 & 2185 & 8.09 & 0.00 & 0.15 & 0.0 & 1.9 & \\
\hline $798 \mathrm{~B}-28 \mathrm{X}-4,31-35$ & 262.82 & 2218 & 9.15 & 0.94 & 0.59 & 10.3 & 6.4 & 1.6 \\
\hline 798B-28X-5, 29-31 & 264.09 & 2227 & 9.31 & 0.78 & 0.53 & 8.4 & 5.7 & 1.5 \\
\hline $798 \mathrm{~B}-28 \mathrm{X}-6,27-33$ & 265.35 & 2236 & 7.46 & 0.55 & 0.39 & 7.4 & 5.2 & 1.4 \\
\hline $798 \mathrm{~B}-29 \mathrm{X}-1,31-33$ & 268.55 & 2258 & 11.30 & 1.63 & 0.98 & 14.4 & 8.7 & 1.7 \\
\hline $798 \mathrm{~B}-29 \mathrm{X}-3,30-35$ & 270.94 & 2275 & 7.70 & 0.48 & 0.10 & 6.2 & 1.3 & 4.8 \\
\hline $798 \mathrm{~B}-29 \mathrm{X}-3,31-33$ & 270.95 & 2275 & 7.69 & 0.52 & 0.12 & 6.7 & 1.5 & 4.5 \\
\hline $798 \mathrm{~B}-29 \mathrm{X}-5,31-33$ & 273.36 & 2292 & 9.25 & 0.85 & 0.54 & 9.2 & 5.8 & 1.6 \\
\hline $798 \mathrm{~B}-30 \mathrm{X}-2,100-102$ & 279.70 & 2337 & 13.68 & 2.13 & 1.18 & 15.6 & 8.6 & 1.8 \\
\hline $798 \mathrm{~B}-30 \mathrm{X}-3,100-102$ & 281.20 & 2347 & 13.62 & 2.11 & 0.72 & 15.5 & 5.3 & 2.9 \\
\hline $798 \mathrm{~B}-30 \mathrm{X}-5,100-102$ & 284.20 & 2368 & 16.82 & 3.55 & 1.72 & 21.1 & 10.2 & 2.1 \\
\hline 798B-30X-6, 100-102 & 285.70 & 2379 & 8.94 & 0.89 & 0.55 & 10.0 & 6.1 & 1.6 \\
\hline $798 \mathrm{~B}-31 \mathrm{X}-2,30-35$ & 288.03 & 2395 & 8.57 & 0.39 & 0.32 & 4.6 & 3.7 & 1.2 \\
\hline $798 \mathrm{~B}-31 \mathrm{X}-4,55-60$ & 290.99 & 2416 & 10.13 & 0.38 & 1.28 & 3.8 & 12.6 & 0.3 \\
\hline $798 \mathrm{~B}-31 \mathrm{X}-6,30-35$ & 293.48 & 2434 & 9.18 & 0.88 & 0.92 & 9.6 & 10.0 & 1.0 \\
\hline $798 \mathrm{~B}-31 \mathrm{X}-7,86-88$ & 295.36 & 2447 & 9.41 & 0.88 & 0.45 & 9.4 & 4.8 & 2.0 \\
\hline $798 \mathrm{~B}-31 \mathrm{X}-8,30-35$ & 296.21 & 2453 & 9.33 & 0.76 & 0.71 & 8.1 & 7.6 & 1.1 \\
\hline $798 \mathrm{~B}-32 \mathrm{X}-2,40-45$ & 297.54 & 2462 & 9.41 & 0.64 & 0.72 & 6.8 & 7.7 & 0.9 \\
\hline $798 \mathrm{~B}-32 \mathrm{X}-3,20-22$ & 298.60 & 2470 & 8.63 & 0.54 & 0.55 & 6.3 & 6.4 & 1.0 \\
\hline $798 \mathrm{~B}-32 \mathrm{X}-4,70-75$ & 300.22 & 2488 & 5.13 & 0.31 & 0.12 & 6.1 & 2.4 & 2.5 \\
\hline $798 \mathrm{~B}-32 \mathrm{X}-5,20-22$ & 301.04 & 2498 & 5.29 & 0.30 & 0.18 & 5.7 & 3.4 & 1.7 \\
\hline $798 \mathrm{~B}-33 \mathrm{X}-1,20-22$ & 305.89 & 2553 & 5.20 & 0.36 & 0.21 & 7.0 & 4.0 & 1.8 \\
\hline $798 \mathrm{~B}-33 \mathrm{X}-4,60-65$ & 310.53 & 2606 & 5.66 & 0.44 & 0.30 & 7.8 & 5.3 & 1.5 \\
\hline $798 \mathrm{~B}-33 \mathrm{X}-5,19-21$ & 311.56 & 2618 & 5.65 & 0.42 & 0.27 & 7.5 & 4.7 & 1.6 \\
\hline $798 \mathrm{~B}-33 \mathrm{X}-6,30-35$ & 313.09 & 2635 & 5.79 & 0.35 & 0.44 & 6.1 & 7.6 & 0.8 \\
\hline $798 \mathrm{~B}-34 \mathrm{X}-1,90-92$ & 316.20 & 2671 & 6.14 & 0.41 & 0.49 & 6.7 & 8.0 & 0.8 \\
\hline $798 \mathrm{~B}-34 \mathrm{X}-2,84-89$ & 317.64 & 2687 & 5.90 & 0.47 & 0.21 & 7.9 & 3.6 & 2.2 \\
\hline $798 \mathrm{~B}-34 \mathrm{X}-4,5-10$ & 319.85 & 2713 & 5.89 & 0.47 & 0.25 & 7.9 & 4.3 & 1.8 \\
\hline $798 \mathrm{~B}-34 \mathrm{X}-5,39-41$ & 321.69 & 2734 & 7.09 & 0.88 & 0.43 & 12.4 & 6.1 & 2.0 \\
\hline 798B-34X-6, 24-29 & 323.04 & 2749 & 7.20 & 1.01 & 0.49 & 14.0 & 6.8 & 2.1 \\
\hline 798B-35X-3, 39-41 & 328.39 & 2810 & 7.81 & 0.70 & 0.49 & 8.9 & 6.3 & 1.4 \\
\hline $798 \mathrm{~B}-35 \mathrm{X}-4,40-45$ & 329.90 & 2827 & 8.24 & 0.79 & 0.63 & 9.6 & 7.7 & 1.3 \\
\hline $798 B-35 X-5,40-42$ & 331.40 & 2845 & 8.43 & 0.97 & 0.69 & 11.5 & 8.2 & 1.4 \\
\hline $798 \mathrm{~B}-35 X-6,60-65$ & 333.10 & 2864 & 7.50 & 0.99 & 0.58 & 13.2 & 7.7 & 1.7 \\
\hline $798 \mathrm{~B}-35 \mathrm{X}-7,5-7$ & 334.05 & 2875 & 7.11 & 0.78 & 0.43 & 11.0 & 6.1 & 1.8 \\
\hline $798 \mathrm{~B}-36 \mathrm{X}-1,80-82$ & 335.40 & 2890 & 8.00 & 1.17 & 0.49 & 14.6 & 6.1 & 2.4 \\
\hline $798 B-36 X-2,20-22$ & 336.30 & 2901 & 9.41 & 1.19 & 0.76 & 12.6 & 8.1 & 1.6 \\
\hline $798 \mathrm{~B}-36 \mathrm{X}-3,14-16$ & 337.74 & 2917 & 9.10 & 1.26 & 0.58 & 13.9 & 6.4 & 2.2 \\
\hline $798 \mathrm{~B}-37 \mathrm{X}-1,60-62$ & 344.90 & 2999 & 9.03 & 1.06 & 0.55 & 11.7 & 6.1 & 1.9 \\
\hline $798 \mathrm{~B}-37 \mathrm{X}-2,46-48$ & 346.26 & 3014 & 8.70 & 1.17 & 0.14 & 13.5 & 1.6 & 8.4 \\
\hline $798 \mathrm{~B}-38 \mathrm{X}-1,17-19$ & 354.07 & 3104 & 7.24 & 0.59 & 0.41 & 8.1 & 5.6 & 1.5 \\
\hline $798 B-38 X-2,40-42$ & 355.80 & 3123 & 7.57 & 0.77 & 0.00 & 10.2 & 0.0 & \\
\hline $798 B-38 X-4,31-33$ & 358.71 & 3157 & 6.46 & 0.63 & 0.39 & 9.8 & 6.1 & 1.6 \\
\hline
\end{tabular}


Table 1 (continued).

\begin{tabular}{|c|c|c|c|c|c|c|c|c|}
\hline $\begin{array}{l}\text { Hole, core, section, } \\
\text { interval }(\mathrm{cm})\end{array}$ & $\begin{array}{l}\text { Depth } \\
\text { (mbsf) }\end{array}$ & $\begin{array}{l}\text { Age } \\
(\mathrm{Ma})\end{array}$ & $\begin{array}{c}\text { Bulk } \\
\text { accumulation }\end{array}$ & Quartz & $\begin{array}{l}\text { Feldspar } \\
\mathrm{g} / \mathrm{cm}^{2} / \mathrm{k} . \mathrm{y}\end{array}$ & $\begin{array}{l}\text { Quartz } \\
\text { (wt\%) }\end{array}$ & $\begin{array}{l}\text { Feldspar } \\
(w t \%)\end{array}$ & $\begin{array}{c}\text { Quartz/Feldspar } \\
\text { ratio }\end{array}$ \\
\hline $798 B-38 X-5,24-26$ & 360.14 & 3173 & 7.41 & 0.77 & 0.37 & 10.4 & 5.0 & 2.1 \\
\hline $798 \mathrm{~B}-39 \mathrm{X}-3,32-34$ & 366.92 & 3251 & 6.80 & 0.60 & 0.29 & 8.8 & 4.3 & 2.1 \\
\hline $798 B-39 X-4,85-87$ & 368.95 & 3274 & 6.52 & 0.59 & 0.32 & 9.1 & 4.9 & 1.9 \\
\hline $798 B-39 X-5,92-94$ & 370.52 & 3292 & 5.13 & 0.53 & 0.23 & 10.3 & 4.5 & 2.3 \\
\hline 798B-39X-6, 127-129 & 372.37 & 3313 & 6.99 & 0.62 & 0.38 & 8.9 & 5.4 & 1.7 \\
\hline $798 \mathrm{~B}-40 \mathrm{X}-1,24-26$ & 373.44 & 3325 & 6.99 & 0.66 & 0.57 & 9.5 & 8.2 & 1.2 \\
\hline $798 B-40 X-2,23-25$ & 374.93 & 3342 & 6.87 & 0.64 & 0.00 & 9.3 & 0.0 & \\
\hline $798 \mathrm{~B}-40 \mathrm{X}-4,131-133$ & 379.01 & 3389 & 6.52 & 0.64 & 0.32 & 9.8 & 4.9 & 2.0 \\
\hline $798 B-41 X-3,60-65$ & 386.40 & 3473 & 6.72 & 0.62 & 0.59 & 9.3 & 8.8 & 1.1 \\
\hline $798 \mathrm{~B}-41 \mathrm{X}-4,60-62$ & 387.85 & 3490 & 7.36 & 0.75 & 0.54 & 10.2 & 7.3 & 1.4 \\
\hline $798 B-41 X-5,61-66$ & 389.32 & 3507 & 8.18 & 0.75 & 0.47 & 9.2 & 5.7 & 1.6 \\
\hline $798 \mathrm{~B}-41 \mathrm{X}-6,20-22$ & 390.38 & 3519 & 7.48 & 0.92 & 0.52 & 12.3 & 6.9 & 1.8 \\
\hline $798 \mathrm{~B}-42 \mathrm{X}-2,19-21$ & 394.19 & 3562 & 8.87 & 1.07 & 0.44 & 12.1 & 5.0 & 2.4 \\
\hline $798 \mathrm{~B}-42 \mathrm{X}-3,20-25$ & 395.70 & 3579 & 9.87 & 1.10 & 0.99 & 11.1 & 10.0 & 1.1 \\
\hline $798 B-42 X-4,16-18$ & 397.16 & 3596 & 10.00 & 1.38 & 0.67 & 13.8 & 6.7 & 2.1 \\
\hline $798 \mathrm{~B}-42 \mathrm{X}-7,19-24$ & 401.69 & 3648 & 10.55 & 1.30 & 0.74 & 12.3 & 7.0 & 1.8 \\
\hline $798 \mathrm{~B}-43 \mathrm{X}-2,80-85$ & 404.50 & 3680 & 9.98 & 1.04 & 0.46 & 10.4 & 4.6 & 2.3 \\
\hline $798 B-43 X-4,50-55$ & 407.20 & 3711 & 10.22 & 0.92 & 1.67 & 9.0 & 16.3 & 0.6 \\
\hline $798 B-43 X-6,20-25$ & 409.90 & 3742 & 9.49 & 0.89 & 0.60 & 9.4 & 6.3 & 1.5 \\
\hline $798 B-44 X-2,40-45$ & 413.56 & 3784 & 9.45 & 0.76 & 0.53 & 8.0 & 5.6 & 1.4 \\
\hline $798 \mathrm{~B}-44 \mathrm{X}-7,50-55$ & 420.62 & 3864 & 10.38 & 1.12 & 0.44 & 10.8 & 4.2 & 2.6 \\
\hline $798 \mathrm{~B}-45 \mathrm{X}-7,31-33$ & 428.58 & 3955 & 8.49 & 0.69 & 0.44 & 8.1 & 5.2 & 1.6 \\
\hline $798 \mathrm{~B}-46 \mathrm{X}-3,110-112$ & 435.12 & 4030 & 9.78 & 0.99 & 1.37 & 10.1 & 14,0 & 0.7 \\
\hline $798 B-46 X-4,66-68$ & 436.16 & 4042 & 9.89 & 0.90 & 1.08 & 9.1 & 10.9 & 0.8 \\
\hline $798 B-46 X-5,43-45$ & 437.40 & 4056 & 9.62 & 0.84 & 1.44 & 8.7 & 15.0 & 0.6 \\
\hline $798 \mathrm{~B}-46 \mathrm{X}-6,112-114$ & 439.55 & 4081 & 10.75 & 0.90 & 0.00 & 8.4 & 0.0 & \\
\hline $798 \mathrm{~B}-47 \mathrm{X}-1,26-28$ & 441.06 & 4098 & 8.95 & 0.86 & 1.08 & 9.6 & 12.1 & 0.8 \\
\hline $798 \mathrm{~B}-47 \mathrm{X}-2,7-9$ & 442.35 & 4113 & 9.30 & 1.00 & 1.00 & 10.7 & 10.8 & 1.0 \\
\hline $798 B-47 X-3,74-76$ & 444.50 & 4137 & 8.66 & 0.78 & 0.46 & 9.0 & 5.3 & 1.7 \\
\hline $798 B-47 X-4,44-46$ & 445.69 & 4151 & 8.48 & 0.72 & 0.53 & 8.5 & 6.2 & 1.4 \\
\hline $798 \mathrm{~B}-47 \mathrm{X}-6,80-82$ & 449.02 & 4189 & 8.27 & 0.93 & 0.89 & 11.2 & 10.8 & 1.0 \\
\hline $798 B-48 X-1,44-46$ & 450.84 & 4210 & 10.60 & 1.01 & 0.94 & 9.5 & 8.9 & 1.1 \\
\hline $798 B-47 X-8,0-5$ & 451.20 & 4214 & 8.00 & 0.71 & 0.97 & 8.9 & 12.1 & 0.7 \\
\hline $798 B-48 X-2,21-23$ & 452.11 & 4224 & 9.96 & 1.04 & 0.75 & 10.4 & 7.5 & 1.4 \\
\hline $798 \mathrm{~B}-48 \mathrm{X}-3,104-106$ & 454.44 & 4251 & 10.19 & 1.21 & 1.02 & 11.9 & 10.0 & 1.2 \\
\hline $798 \mathrm{~B}-48 \mathrm{X}-4,62-64$ & 455.52 & 4263 & 11.00 & 1.33 & 0.77 & 12.1 & 7.0 & 1.7 \\
\hline $798 B-48 X-5,97-99$ & 457.37 & 4284 & 11.19 & 1.16 & 1.14 & 10.4 & 10.2 & 1.0 \\
\hline $798 \mathrm{~B}-49 \mathrm{X}-1,63-65$ & 460.71 & 4322 & 11.75 & 1.41 & 2.14 & 12.0 & 18.2 & 0.7 \\
\hline $798 B-49 X-2,110-112$ & 462.62 & 4344 & 9.50 & 0.86 & 0.76 & 9.1 & 8.0 & 1.1 \\
\hline $798 B-49 X-3,76-78$ & 463.75 & 4357 & 10.26 & 0.87 & 1.06 & 8.5 & 10.3 & 0.8 \\
\hline $798 B-49 X-4,112-114$ & 465.56 & 4378 & 9.92 & 0.80 & 0.71 & 8.1 & 7.2 & 1.1 \\
\hline 798B-49X-6, 110-112 & 468.45 & 4411 & 12.10 & 1.56 & 0.91 & 12.9 & 7.5 & 1.7 \\
\hline $798 \mathrm{~B}-49 \mathrm{X}-7,50-55$ & 469.32 & 4421 & 12.00 & 1.92 & 2.21 & 16.0 & 18.4 & 0.9 \\
\hline 798B-50X-1, 75-77 & 470.45 & 4434 & 11.73 & 1.41 & 1.89 & 12.0 & 16.1 & 0.8 \\
\hline $798 \mathrm{~B}-50 \mathrm{X}-2,110-112$ & 472.30 & 4455 & 10.72 & 0.77 & 1.24 & 7.2 & 11.6 & 0.6 \\
\hline $798 B-51 X-3,84-86$ & 483.24 & 4580 & 11.34 & 1.33 & 1.75 & 11.7 & 15.4 & 0.8 \\
\hline $798 \mathrm{~B}-51 \times-4,0-5$ & 483.90 & 4587 & 11.40 & 1.23 & 1.31 & 10.8 & 11.5 & 0.9 \\
\hline 798B-51X-6, 86-88 & 487.76 & 4632 & 11.96 & 1.30 & 1.61 & 10.9 & 13.5 & 0.8 \\
\hline 798B-52X-1, 19-21 & 489.19 & 4648 & 12.11 & 1.27 & 2.01 & 10.5 & 16.6 & 0.6 \\
\hline $798 B-52 X-2,17-19$ & 490.65 & 4665 & 11.53 & 1.07 & 0.96 & 9.3 & 8.3 & 1.1 \\
\hline 798B-52X-3, 93-95 & 492.89 & 4690 & 11.71 & 1.37 & 0.73 & 11.7 & 6.2 & 1.9 \\
\hline 798B-52X-4, 94-96 & 494.39 & 4707 & 11.51 & 1.23 & 0.87 & 10.7 & 7.6 & 1.4 \\
\hline 798B-52X-5, 91-93 & 495.84 & 4724 & 10.92 & 1.17 & 1.05 & 10.7 & 9.6 & 1.1 \\
\hline $798 \mathrm{~B}-52 \mathrm{X}-6,40-42$ & 496.82 & 4735 & 11.03 & 1.11 & 1.28 & 10.1 & 11.6 & 0.9 \\
\hline $798 \mathrm{~B}-53 \mathrm{X}-1,121-123$ & 499.91 & 4770 & 11.55 & 1.16 & 0.75 & 10.0 & 6.5 & 1.5 \\
\hline $798 B-53 X-3.0-5$ & 501.70 & 4791 & 11.30 & 0.97 & 1.11 & 8.6 & 9.8 & 0.9 \\
\hline $798 \mathrm{~B}-53 \mathrm{X}-4,56-58$ & 503.76 & 4814 & 11.40 & 1.14 & 0.91 & 10.0 & 8.0 & 1.3 \\
\hline $798 B-53 X-5,8-10$ & 504.78 & 4826 & 11.51 & 1.05 & 0.92 & 9.1 & 8.0 & 1.1 \\
\hline $798 \mathrm{~B}-54 \mathrm{X}-1,54-56$ & 508.78 & 4872 & 11.20 & 0.93 & 1.36 & 8.3 & 12.1 & 0.7 \\
\hline $798 B-54 X-2,56-58$ & 510.11 & 4887 & 10.26 & 0.70 & 1.15 & 6.8 & 11.2 & 0.6 \\
\hline $798 \mathrm{~B}-54 \mathrm{X}-3,123-125$ & 512.03 & 4909 & 10.91 & 0.96 & 1.01 & 8.8 & 9.3 & 1.0 \\
\hline $798 \mathrm{~B}-54 \mathrm{X}-4,131-133$ & 513.42 & 4925 & 11.67 & 1.14 & 0.95 & 9.8 & 8.1 & 1.2 \\
\hline $798 B-54 X-5,92-94$ & 514.40 & 4936 & 11.61 & 1.04 & 1.93 & 9.0 & 16.6 & 0.5 \\
\hline $798 B-54 X-7,0-5$ & 516.23 & 4957 & 11.50 & 1.12 & 1.18 & 9.7 & 10.3 & 0.9 \\
\hline 798B-54X-8, 55-60 & 518.04 & 4978 & 11.50 & 1.22 & 0.70 & 10.6 & 6.1 & 1.7 \\
\hline
\end{tabular}


Table 2. Composition of the clay mineral fraction as relative percentage of illite, chlorite, kaolinite, and smectite, and kaolinite/illite, and smectite/illite ratios.

\begin{tabular}{|c|c|c|c|c|c|c|c|}
\hline $\begin{array}{l}\text { Hole, core, section, } \\
\text { interval }(\mathrm{cm})\end{array}$ & $\begin{array}{l}\text { Depth } \\
\text { (mbsf) }\end{array}$ & $\begin{array}{l}\text { Illite } \\
(\%)\end{array}$ & $\begin{array}{l}\text { Chlorite } \\
(\%)\end{array}$ & $\begin{array}{l}\text { Kaolinite } \\
(\%)\end{array}$ & $\begin{array}{l}\text { Smectite } \\
(\%)\end{array}$ & $\begin{array}{l}\text { Kaolinite/Illite } \\
\text { ratio }\end{array}$ & $\begin{array}{c}\text { Smectite/Illite } \\
\text { ratio }\end{array}$ \\
\hline $798 \mathrm{~A}-1 \mathrm{H}-3,37-39$ & 3.37 & 79 & 11 & 10 & 0 & 0.12 & 0.00 \\
\hline $798 \mathrm{~A}-1 \mathrm{H}-5,2-7$ & 6.02 & 82 & 9 & 9 & 0 & 0.11 & 0.00 \\
\hline $798 \mathrm{~A}-2 \mathrm{H}-1,129-131$ & 10.59 & 87 & 7 & 6 & 0 & 0.07 & 0.00 \\
\hline $798 \mathrm{C}-2 \mathrm{H}-4,53-55$ & 11.38 & 76 & 24 & 0 & 0 & 0.00 & 0.00 \\
\hline $798 \mathrm{C}-2 \mathrm{H}-4,71-73$ & 11.57 & 71 & 12 & 9 & 8 & 0.12 & 0.11 \\
\hline $798 \mathrm{C}-2 \mathrm{H}-4,73-75$ & 11.60 & 68 & 15 & 9 & 8 & 0.12 & 0.11 \\
\hline $798 \mathrm{C}-2 \mathrm{H}-4,125-127$ & 12.16 & 76 & 15 & 9 & 0 & 0.12 & 0.00 \\
\hline $798 \mathrm{~A}-2 \mathrm{H}-3,25-30$ & 12.55 & 66 & 14 & 12 & 8 & 0.18 & 0.12 \\
\hline $798 \mathrm{~A}-3 \mathrm{H}-6,44-49$ & 26.24 & 82 & 11 & 7 & 0 & 0.09 & 0.00 \\
\hline $798 \mathrm{~A}-5 \mathrm{H}-5,48-50$ & 43.22 & 75 & 25 & 0 & 0 & 0.00 & 0.00 \\
\hline $798 \mathrm{~A}-6 \mathrm{H}-2,82-87$ & 48.56 & 85 & 15 & 0 & 0 & 0.00 & 0.00 \\
\hline $798 \mathrm{~A}-6 \mathrm{H}-3,7-9$ & 49.27 & 74 & 15 & 11 & 0 & 0.15 & 0.00 \\
\hline $798 \mathrm{~A}-6 \mathrm{H}-3,18-20$ & 49.39 & 73 & 27 & 0 & 0 & 0.00 & 0.00 \\
\hline $798 \mathrm{~A}-6 \mathrm{H}-3,28-30$ & 49.49 & 76 & 24 & 0 & 0 & 0.00 & 0.00 \\
\hline $798 \mathrm{~A}-6 \mathrm{H}-3,35-37$ & 49.57 & 76 & 24 & 0 & 0 & 0.00 & 0.00 \\
\hline $798 \mathrm{~A}-7 \mathrm{H}-4,24-26$ & 60.48 & 75 & 9 & 9 & 6 & 0.11 & 0.09 \\
\hline $798 \mathrm{~A}-7 \mathrm{H}-4,123-127$ & 61.66 & 85 & 7 & 8 & 0 & 0.09 & 0.00 \\
\hline 798A-7H-5, 131-133 & 63.25 & 79 & 10 & 11 & 0 & 0.14 & 0.00 \\
\hline $798 \mathrm{~A}-10 \mathrm{H}-1,44-46$ & 84.89 & 80 & 7 & 7 & 6 & 0.09 & 0.08 \\
\hline $798 \mathrm{~A}-10 \mathrm{H}-3,83-85$ & 88.33 & 77 & 23 & 0 & 0 & 0.00 & 0.00 \\
\hline $798 \mathrm{~A}-10 \mathrm{H}-5,39-41$ & 90.84 & 73 & 15 & 12 & 0 & 0.16 & 0.00 \\
\hline $798 \mathrm{~A}-10 \mathrm{H}-7,35-37$ & 93.79 & 80 & 9 & 11 & 0 & 0.13 & 0.00 \\
\hline $798 \mathrm{~A}-11 \mathrm{H}-3,94-96$ & 98.09 & 72 & 13 & 9 & 7 & 0.12 & 0.09 \\
\hline $798 \mathrm{~A}-11 \mathrm{H}-4,2-4$ & 98.62 & 80 & 11 & 9 & 0 & 0.11 & 0.00 \\
\hline $798 \mathrm{~A}-11 \mathrm{H}-3,146-148$ & 98.63 & 78 & 22 & 0 & 0 & 0.00 & 0.00 \\
\hline $798 \mathrm{~A}-12 \mathrm{H}-8,0-2$ & 114.30 & 77 & 14 & 9 & 0 & 0.11 & 0.00 \\
\hline $798 \mathrm{~A}-12 \mathrm{H}-8,23-25$ & 114.57 & 81 & 11 & 8 & 0 & 0.10 & 0.00 \\
\hline $798 \mathrm{~A}-12 \mathrm{H}-8,52-54$ & 114.90 & 78 & 14 & 8 & 0 & 0.10 & 0.00 \\
\hline $798 \mathrm{~A}-12 \mathrm{H}-8,62-64$ & 115.02 & 80 & 14 & 6 & 0 & 0.08 & 0.00 \\
\hline $798 \mathrm{~A}-12 \mathrm{H}-8,70-72$ & 115.11 & 77 & 18 & 5 & 0 & 0.07 & 0.00 \\
\hline $798 \mathrm{~A}-12 \mathrm{H}-8,75-77$ & 115.17 & 76 & 15 & 9 & 0 & 0.12 & 0.00 \\
\hline $798 \mathrm{~A}-14 \mathrm{H}-2,112-114$ & 125.84 & 82 & 7 & 7 & 4 & 0.09 & 0.04 \\
\hline $798 \mathrm{~A}-14 \mathrm{H}-3,118-122$ & 127.41 & 84 & 9 & 7 & 0 & 0.09 & 0.00 \\
\hline 798B-14H-5, 39-41 & 129.66 & 85 & 8 & 7 & 0 & 0.09 & 0.00 \\
\hline $798 \mathrm{~A}-14 \mathrm{H}-8,66-68$ & 134.33 & 87 & 7 & 6 & 0 & 0.07 & 0.00 \\
\hline $798 \mathrm{~A}-15 \mathrm{H}-3,103-107$ & 137.04 & 75 & 14 & 11 & 0 & 0.15 & 0.00 \\
\hline $798 \mathrm{~B}-15 \mathrm{H}-7,75-77$ & 142.79 & 88 & 7 & 5 & 0 & 0.06 & 0.00 \\
\hline 798B-16X-5, 57-59 & 149.18 & 85 & 15 & 0 & 0 & 0.00 & 0.00 \\
\hline 798B-17X-7, 39-41 & 161.71 & 83 & 9 & 8 & 0 & 0.10 & 0.00 \\
\hline $798 \mathrm{~B}-18 \mathrm{X}-2,30-35$ & 163.70 & 79 & 21 & 0 & 0 & 0.00 & 0.00 \\
\hline $798 \mathrm{~B}-20 \mathrm{X}-3,40-42$ & 184.79 & 77 & 8 & 7 & 7 & 0.09 & 0.10 \\
\hline 798B-21X-5, 35-37 & 197.35 & 77 & 10 & 7 & 6 & 0.10 & 0.08 \\
\hline 798B-22X-3, 71-73 & 204.47 & 81 & 19 & 0 & 0 & 0.00 & 0.00 \\
\hline 798B-23X-3, 25-27 & 213.60 & 70 & 16 & 0 & 14 & 0.00 & 0.20 \\
\hline 798B-24X-3, 49-51 & 223.50 & 72 & 9 & 9 & 10 & 0.12 & 0.14 \\
\hline 798B-25X-5, 60-62 & 236.30 & 79 & 21 & 0 & 0 & 0.00 & 0.00 \\
\hline 798B-27X-3, 38-40 & 252.38 & 79 & 21 & 0 & 0 & 0.00 & 0.00 \\
\hline 798B-28X-2, 27-32 & 260.52 & 83 & 10 & 7 & 0 & 0.09 & 0.00 \\
\hline 798B-29X-7, 35-37 & 277.74 & 84 & 16 & 0 & 0 & 0.00 & 0.00 \\
\hline $798 \mathrm{~B}-30 \mathrm{X}-4,100-105$ & 282.70 & 83 & 10 & 7 & 0 & 0.08 & 0.00 \\
\hline 798B-31X-1, 30-32 & 286.73 & 68 & 0 & 21 & 11 & 0.31 & 0.16 \\
\hline 798B-32X-1, 129-131 & 297.59 & 80 & 11 & 9 & 0 & 0.12 & 0.00 \\
\hline 798B-33X-3, 79-81 & 309.53 & 80 & 11 & 9 & 0 & 0.11 & 0.00 \\
\hline 798B-34X-3, 87-89 & 319.17 & 84 & 16 & 0 & 0 & 0.00 & 0.00 \\
\hline 798B-35X-1, 15-17 & 325.15 & 83 & 10 & 7 & 0 & 0.09 & 0.00 \\
\hline $798 \mathrm{~B}-37 \mathrm{X}-3,44-46$ & 347.74 & 74 & 9 & 7 & 10 & 0.09 & 0.13 \\
\hline $798 \mathrm{~B}-38 \mathrm{X}-3,31-33$ & 357.21 & 71 & 8 & 7 & 14 & 0.09 & 0.19 \\
\hline $798 B-39 X-1,14-16$ & 363.74 & 82 & 10 & 8 & 0 & 0.09 & 0.00 \\
\hline $798 \mathrm{~B}-42 \mathrm{X}-5,140-142$ & 399.90 & 60 & 9 & 8 & 22 & 0.13 & 0.37 \\
\hline 798B-44X-1, 60-62 & 412.45 & 72 & 8 & 8 & 13 & 0.11 & 0.18 \\
\hline $798 \mathrm{~B}-44 \mathrm{X}-1,105-110$ & 412.93 & 47 & 6 & 5 & 42 & 0.11 & 0.88 \\
\hline $798 \mathrm{~B}-45 \mathrm{X}-5,132-134$ & 429.24 & 31 & 5 & 5 & 59 & 0.16 & 1.90 \\
\hline $798 \mathrm{~B}-46 \mathrm{X}-2,131-133$ & 433.94 & 41 & 4 & 6 & 48 & 0.14 & 1.18 \\
\hline $798 \mathrm{~B}-47 \mathrm{X}-5,4-45$ & 447.23 & 39 & 6 & 6 & 49 & 0.16 & 1.26 \\
\hline $798 \mathrm{~B}-48 \mathrm{X}-2,28-30$ & 452.18 & 43 & 5 & 6 & 46 & 0.13 & 1.06 \\
\hline $798 B-48 X-4,62-64$ & 455.52 & 30 & 4 & 4 & 63 & 0.12 & 2.13 \\
\hline $798 \mathrm{~B}-49 \mathrm{X}-3,131-133$ & 464.45 & 62 & 9 & 7 & 23 & 0.12 & 0.36 \\
\hline $798 \mathrm{~B}-49 \mathrm{X}-7,23-25$ & 469.34 & 46 & 7 & 6 & 41 & 0.13 & 0.90 \\
\hline $798 \mathrm{~B}-50 \mathrm{X}-3,132-135$ & 474.02 & 42 & 14 & 0 & 44 & 0.00 & 1.06 \\
\hline 798B-51X-6, 21-24 & 487.11 & 32 & 4 & 3 & 61 & 0.10 & 1.89 \\
\hline $798 \mathrm{~B}-52 \mathrm{X}-6,128-130$ & 497.79 & 51 & 7 & 6 & 36 & 0.12 & 0.71 \\
\hline
\end{tabular}




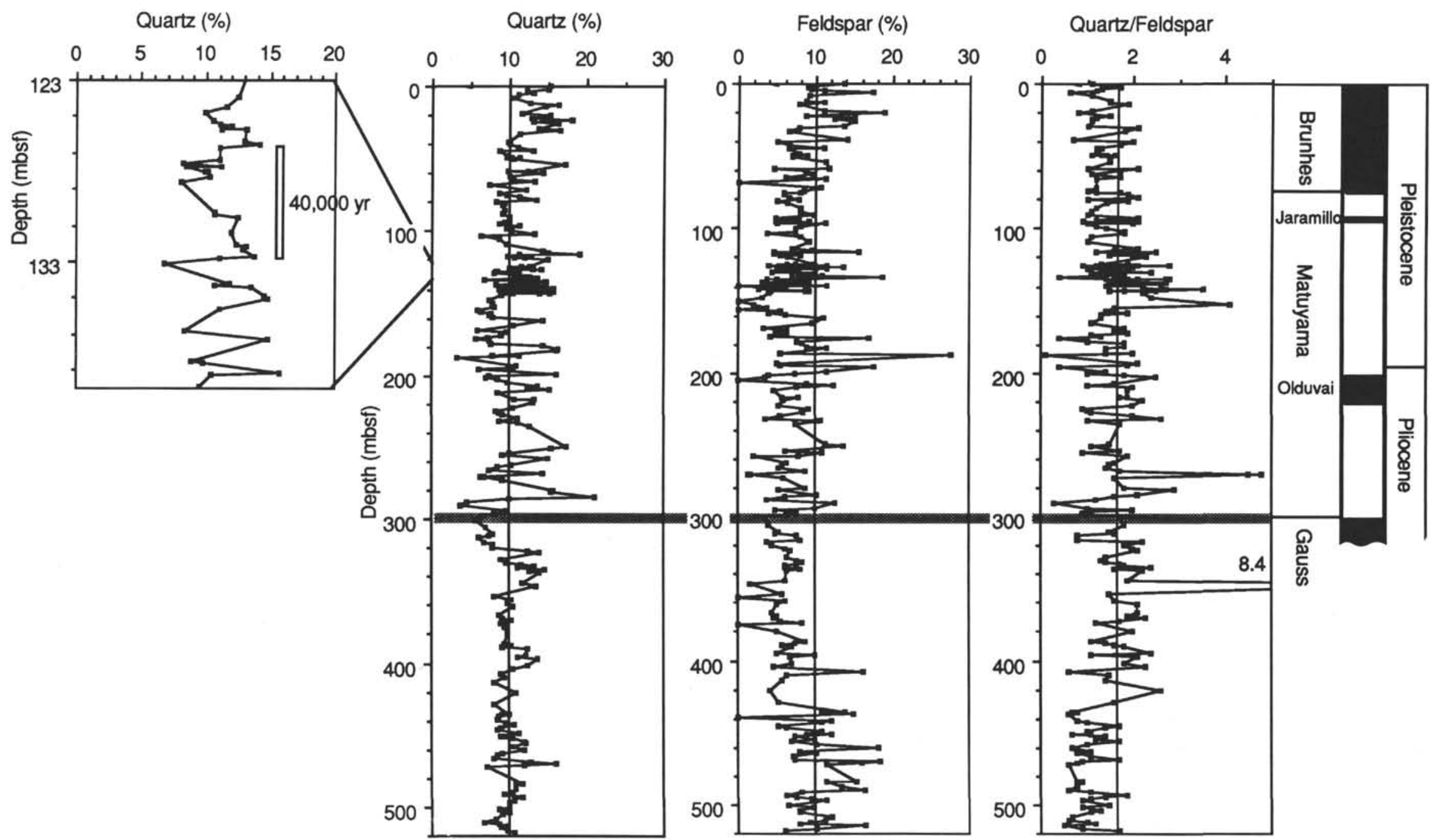

Figure 3. Quartz and feldspar contents and quartz/feldspar ratios of bulk sediment vs. depth at Site 798. Magnetic reversal column as far as Gauss/Matuyama boundary (Ingle, Suyehiro, von Breymann, et al., 1990). 


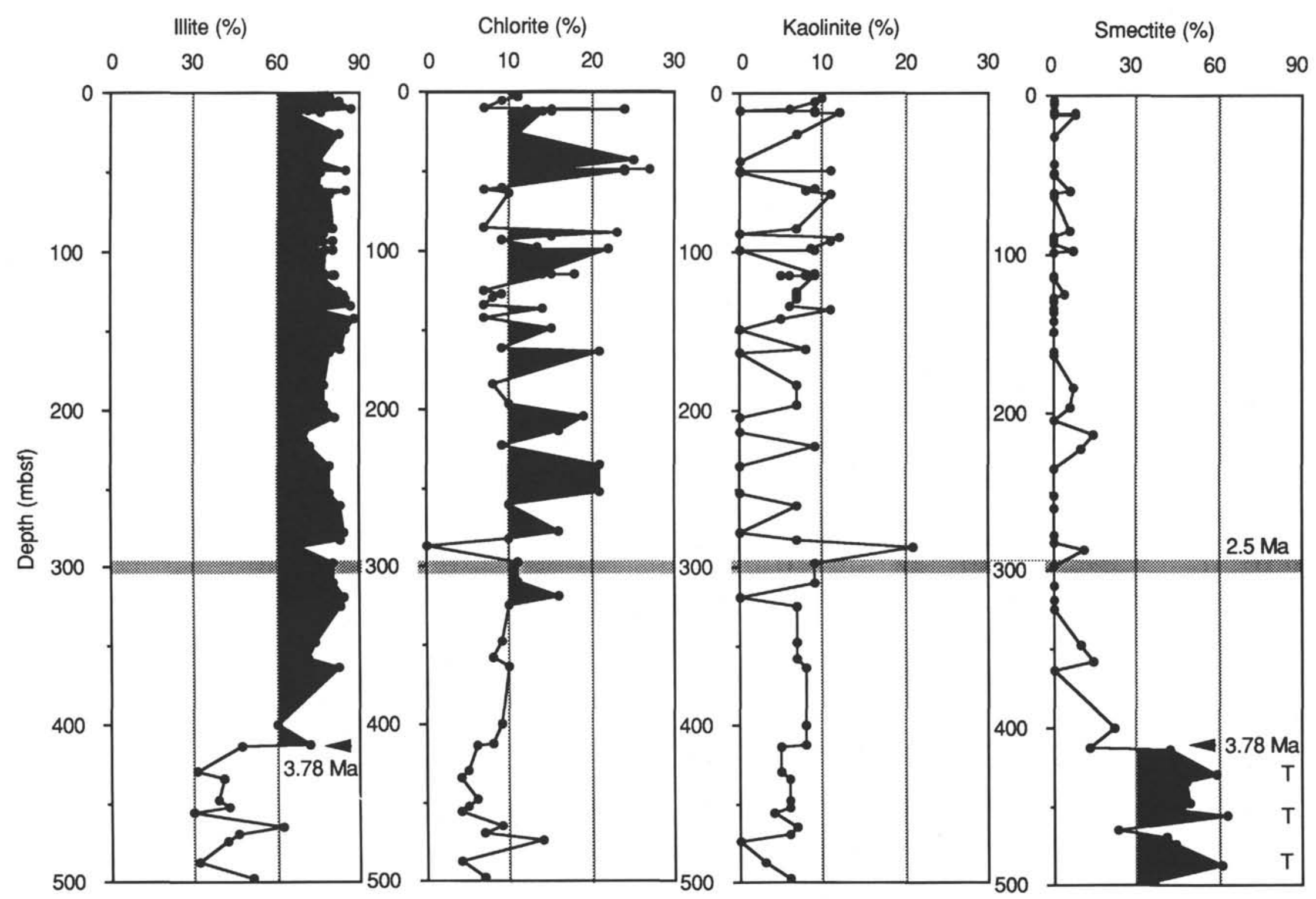

Figure 4. Composition of the clay mineralogy referred to $100 \mathrm{wt} \%$ clay fraction of Site $798 ; \mathrm{T}=$ attributed to turbidity currents. 
k.y. ${ }^{-1}$ and a mean value of $0.77 \mathrm{~g} \mathrm{~cm}^{-2} \mathrm{k.y} .^{-1}$, if the time interval increases from 6 to $4.3 \mathrm{Ma}$ ). The following unit from 4.3 to $2.4 \mathrm{Ma}$ represents a period of relatively constant, low feldspar accumulation with a mean rate of $0.51 \mathrm{~g} \mathrm{~cm}^{-2} \mathrm{k} . \mathrm{y}^{-1}$ (range: $0-1.44 \mathrm{~g} \mathrm{~cm}^{-2} \mathrm{k} . \mathrm{y}{ }^{-1}$ ), The last $2.4 \mathrm{Ma}$ are characterized by a different pattern, revealing at the base a distinct increase of feldspar accumulation rates (mean value: $0.82 \mathrm{~g} \mathrm{~cm}^{-2} \mathrm{k} . \mathrm{y} .{ }^{-1}$; range: $0-3.04 \mathrm{~g} \mathrm{~cm}^{-2} \mathrm{k} . \mathrm{y}^{-1}$ ) with a higher amplitude and a more short-term variation (Fig. 5). Between 2.4 and $1.1 \mathrm{Ma}$, and during the last $0.4 \mathrm{Ma}$, maximum rates of $1.5-2.5 \mathrm{~g} \mathrm{~cm}^{-2}$ k.y. ${ }^{-1}$ are typical.

\section{DISCUSSION}

In general, the accumulation rates and composition of the siliciclastic material (i.e., quartz, feldspars, and clay minerals) may allow us (1) to reveal different source areas (i.e., the Asian continent and/or the Japanese islands), (2) to distinguish different transport mechanisms (i.e., fluvial or eolian), and (3) to reconstruct the climate of the source areas (e.g., Thiede, 1979; Sarnthein et al., 1981, 1982; Rea and Janecek, 1982; Stein, 1985a, 1985b; Chamley, 1989).

Accumulation rates and composition of the siliciclastic material as shown in Figures 3-6, demonstrate long-term (i.e., the order of $100,000 \mathrm{yr}$ ) and short-term (i.e., about $40,000 \mathrm{yr}$ ) fluctuations, which are probably controlled by climate- and oceanographic-induced changes as well as changes in transport mechanisms. Furthermore, they seem to fit into orbital-forced processes, as spectral analyses show significant frequencies at 103, 41, 27, 19, 16, 12, and $10 \mathrm{k} . \mathrm{y}$. (Dunbar et al., this volume; Föllmi et al., this volume).

During (?latest Miocene/) earliest Pliocene, the terrigenous sediment input was characterized by increased flux rates of feldspar, low quartz/feldspar-ratios, and maximum contents of smectite, which is distinctly different from the late Pliocene/Pleistocene record. Furthermore, in the same interval, well-rounded glauconites grains and coarse-grained quartz were recorded, which were interpreted as allochthonous components (Ingle, Suyehiro, von Breymann, et al., 1990). These characteristics imply distinctly different transport mechanisms and probably different source areas of the siliciclastic material in comparison to the younger sediments. We attribute these changes to turbidity currents as the major transport mechanism. These sediments were probably deposited prior to significant uplift of the present-day Oki Ridge. The uplift of the Oki Ridge during the late Pliocene/Pleistocene is indicated by the disappearance of coarsegrained (turbiditic) terrigenous sediments and the decrease in accumulation rates (Fig. 5). Between 3.78 and $2.4 \mathrm{Ma}$ a gradual increase in the amounts of illite, chlorite, and kaolinite is documented until at 2.4 Ma the values already reach their present-day level. This development is interpreted as the response to a gradual change to dominantly physical weathering due to more arid climatic conditions.

The next distinct change occurs between 2.6 and $2.4 \mathrm{Ma}$. The late Pliocene/Pleistocene record is characterized by distinctly higher quartz and feldspar accumulation rates (Fig. 5). This change is interpreted as a climate-controlled increase in eolian sediment supply at Oki Ridge at that time. The eolian transport mechanism of terrigenous sediments is also corroborated by the dominance of silt-sized quartz grains as based on smear-slide descriptions (Ingle, Suyehiro, von Breymann, et al., 1990). Furthermore, this increase is almost synchronous with the major expansion of deserts and deposition of loess in China (Kukla, 1987; Tungsheng, 1988). The increase in eolian sediment supply is accompanied by an increase of (marine) organic carbon and biogenic opal, due to an increase in surface water productivity (Stein and Stax, this volume). These paleoenvironmental changes were probably caused by intensified oceanic and atmospherical circulation and aridification in Asia triggered by the development of major Northern Hemisphere glaciation near 2.5 Ma (e.g., Shackleton et al., 1984). Similar changes have been recorded in other parts of the global ocean, such as the tropical/subtropical Atlantic (e.g., Stein 1985a, 1985b; Ruddiman and Janecek, 1989) or southwestern Pacific (e.g., Dersch and Stein, in press).

In general, the upper Pliocene/Pleistocene record at Site 798 reflects a depositional sequence characterized by visible long- and short-term cyclic changes in sediment flux and composition, whereas short-term $(40,000 \mathrm{yr})$ cycles can be overprinted by the long-term cycles $(100,000 \mathrm{yr}$; Föllmi et al., this volume). Since there was no detailed $\delta^{18} \mathrm{O}$ record available until now, interpretation of the cyclicity in terms of paleoenvironmental conditions is still highly preliminary. The uplift of the Oki Ridge during the late Pliocene/Pleistocene as well as the intensified volcanic activity on the Japanese islands since about 1.6 Ma (Ingle, Suyehiro, von Breymann, et al., 1990) must also be considered as a possible overprinting factor to the cycles. Low accumulation rates of terrigenous matter occur between 0.7 and 0.9 , 1.3 and 1.4, and 1.7 and $1.9 \mathrm{Ma}$ (Fig. 5), which may suggest a reduced eolian sediment supply resulting from more humid climatic conditions. Furthermore, there is an increase in carbonate accumulation since $1.9 \mathrm{Ma}$ (cf. Stein and Stax, this volume), probably resulting from a gradual emergence of Oki Ridge above the early Pleistocene CCD (Ingle, Suyehiro, von Breymann, et al., 1990). Between 1.7 and 1.9 $\mathrm{Ma}$, i.e., during the Olduvai paleomagnetic event, a phase of prolonged warm and relatively humid climate is also indicated by sedimentological and paleobotanical evidence in China, e.g., by a series of dense polygenetic soils within the Chinese loess soil sequence (Kukla, 1987). There is also a consistent pattern of loess deposition during glacial maxima and the formation of soil horizons during interglacial periods for the last $1 \mathrm{Ma}$ (Heller and Liu, 1984). Maxima in the amounts of terrigenous sediments determined by logging data are also interpreted as an increased Asian eolian dust supply associated with glacial maxima (de Menocal et al., this volume; with aluminum as proxy). The composition of the clay fraction with illite and chlorite as dominant clay minerals in the upper Pliocene/Pleistocene record at Site 798 reflects dominantly physical weathering due to more arid climate conditions in the source area of the terrigenous material, too.

The short-term cycles in the Site 798 sediments show two types of periods: a period equivalent to "Milankovitch-type" cyclicity of about 40,000 yr (5-6 $\mathrm{m}$ in thickness) (Fig. 3; de Menocal et al., this volume) and within these cycles, dark/light subcycles of 1000-12,000 $\mathrm{yr}$ that are characterized by distinct changes in carbonate and organic carbon contents (Stein and Stax, this volume; Fig. 6). These subcycles demonstrate changes between finely laminated, dark intervals with increased organic carbon and lighter, homogenous to bioturbated intervals with lower organic carbon contents (Föllmi et al., 1990; Stein and Stax, this volume). Whereas the "Milankovitch-type" gla$\mathrm{cial} /$ interglacial cycles show differences in terrigenous sediment flux and composition (Figs. 3 and 5), the investigated dark/light subcycles display no clear differences in their siliciclastic composition (Fig. 6), indicating formation under similar climatic conditions as well as a similar source area. The higher kaolinite value in the upper light part of the cycle shown in Figure 6, on the other hand, may suggest a different (more southern and tropical) source area for the case of this special paleoenvironmental situation. The 40,000 yr cyclicity seems to reflect global influences as climatic changes, whereas the dark/light subcycles might expose regional paleoenvironmental changes, e.g., due to hydrodynamic fluctuations. These short-term subcycles, which are not bound to either glacial or interglacial stages, may be interpreted as phases within glacial or interglacial periods, when oceanographic factors may have caused changes in paleoenvironmental conditions (i.e., anoxic vs. oxic condition; cf. Oba et al., 1991; Stein, 1991; Stein and Stax, this volume). Nevertheless, shipboard and shore-based investigations of these remarkable dark/light cycles (e.g., Ingle, Suyehiro, von Breymann, et al., 1990; Dunbar et al., Föllmi et al., this volume) display a complex relationship between stratigraphic setting, lithology, and the different biogenic and terrigenous sediment parameters when going into detail. This may indicate, that each cycle 


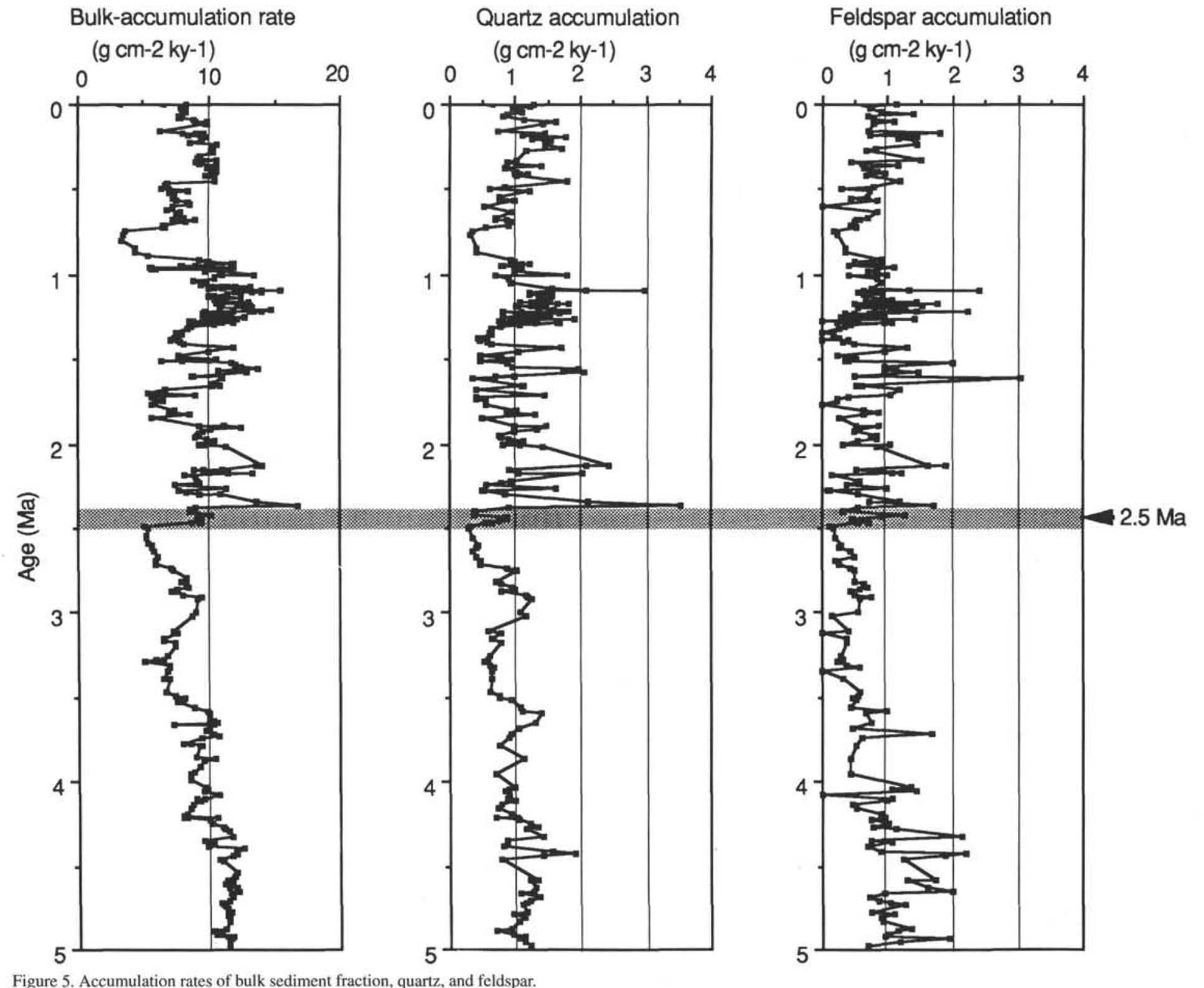


Biogenic

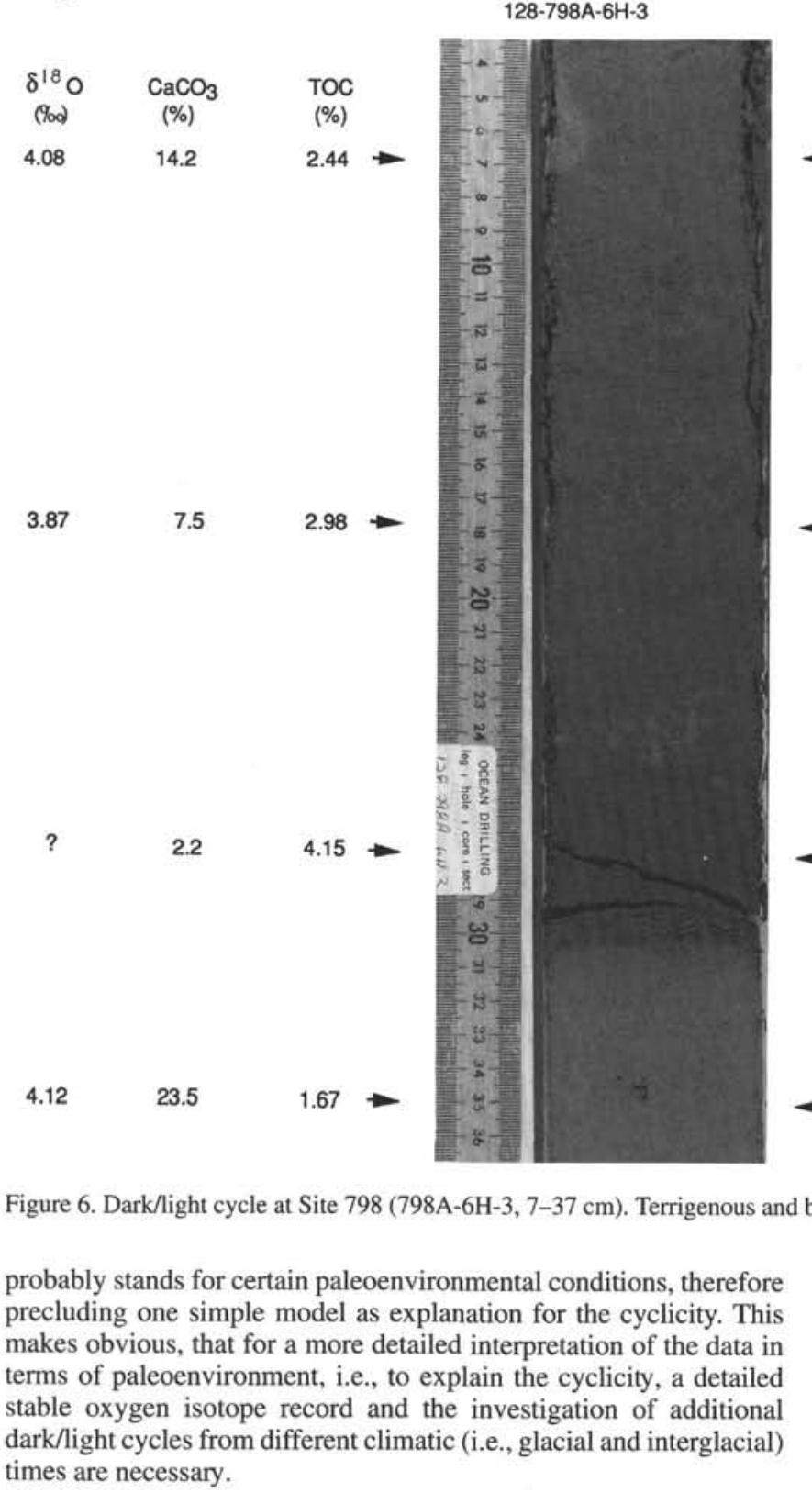

\section{CONCLUSIONS}

Our preliminary results can be summarized as follows:

Sediments from Site 798 are characterized by long-and short-term fluctuations in composition and flux rates of terrigenous material, probably reflecting paleoenvironmental changes in climate, oceanography, and transport mechanisms.

The (?latest Miocene/) earliest Pliocene record is characterized by relatively high amounts of feldspar, low quartz/feldspar ratios, highest smectite values, glauconites, and coarse-grained quartz grains, attributed to a deposition influenced by turbidity currents.

A distinct increase in quartz and feldspar accumulation as well as chlorite contents at about $2.5 \mathrm{Ma}$ point to substantial changes in paleoenvironmental conditions, which is monitored by an increase in both amplitude and period of the fluctuations. This profound change, which can be correlated with the onset of major loess deposition in China, may have been caused by intensified atmos-

\section{Terrigenous}

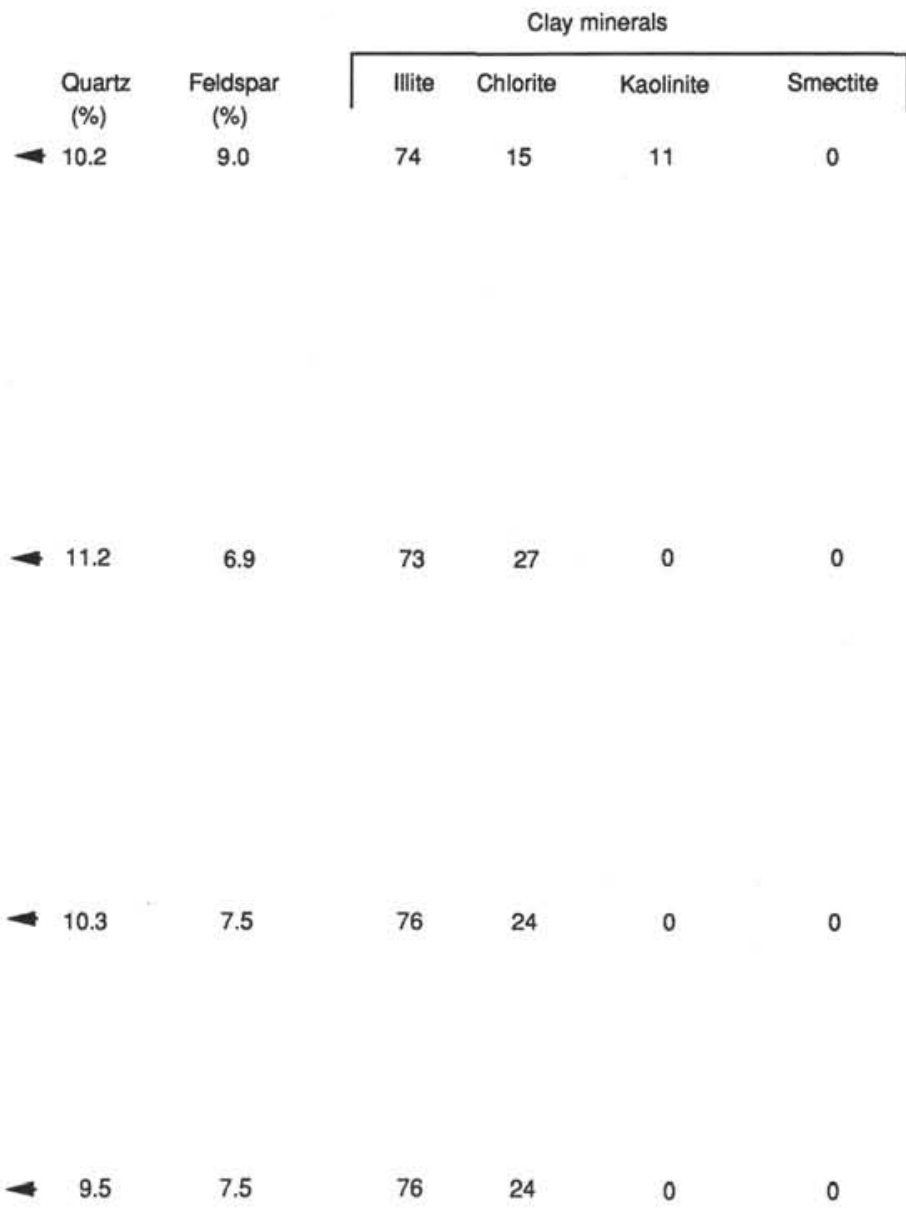




\section{REFERENCES}

Biscaye, P. E., 1965. Mineralogy and sedimentation of recent deep-sea clays in the Atlantic Ocean and adjacent seas and oceans. Geol. Soc. Am. Bull., 76:803-832.

Burbank, D. W., and Jijun, L., 1985. Age and paleoclimatic significance of the loess of Lanzhou, north China. Nature, 316:429-431.

Chamley, H., 1989. Clay Sedimentology: Berlin (Springer Verlag).

Dersch, M., and Stein, R., in press. Paläoklima und paläoozeanische Verhältnisse im SW-Pazifik während der letzten 6 Mill. Jahre (DSDP-Site 594, Chatham Rücken, östlich Neuseeland). Geol. Rndsch.

Elverhøi, A., and Rønningsland, T. M., 1978. Semiquantitative calculation of the relative amounts of kaolinite and chlorite by X-ray diffraction. Mar. Geol., 27:M19-M23.

Emmermann, R., Lauterjung, J., and Stroh, A., 1989. Das Lithostratigraphische Profil der KTB-Vorbohrung, bestimmt durch röntgenographische Phasenanalyse von Bohrkleinen. KTB Rep., 89-3:152-164.

Föllmi, K. B., Alexandrovich, J., Brunner, C. A., Burckle, L. H., Charvet, J., Cramp, A., Demenocal, P., Dunbar, R. B., Grimm, K. A., Holler, P., Ingle, J. C., Isaacs, C. M., Kheradyar, T., Koizumi, I., Matsumoto, R., Nobes, D., Pisciotto, K., Rahnan, A., Stein, R., Tada, R., von Breymann, M., and Scientific Party of Legs 127 and 128, 1990. Paleoceanographic implications from high-frequency dark/light rhythms in the Sea of Japan (ODP Legs 127 and 128). AAPG Circum Pacific Conf. (Abstract)

Gibbs, R. J., 1967. Quantitative X-ray diffraction analyses using clay mineral standard extracted from the samples to be analysed. Clay Miner., 7:79-90.

Heller, F., and Liu, T. S., 1984. Magnetism of chinese loess deposits. Geophys. J. R. Astron. Soc., 77:125-141.

Hovan, S., Rea, D., Pisias, N. G., and Shackleton, N. J., 1989. A direct link between the China loess and marine $\mathrm{O}^{18}$ records: eolian flux to the North Pacific. Nature, 340:296-298.

Ingle, J. C., Jr., Suyehiro, K., von Breyman, M. T., et al., 1990. Proc. ODP, Init. Repts., 128: College Station, TX (Ocean Drilling Program).

Kukla, G., 1987. Loess stratigraphy in central China. Quat. Sci. Rev., 6:191-219.

Kukla, G., Heller, F., Liu, M., Xu, T. C., Liu, S., and An, Z. S., 1988. Pleistocene climates in China dated by magnetic susceptibility. Geology, $16: 811-814$.

Müller, G., 1967. Methods in sedimentary petrology. In Von Engelhardt, W., Füchtbauer, H., and Müller, G. (Eds.), Sedimentary Petrology (Pt. I): Stuttgart (Schweitzerbart'sche Verlagsbuchhandlung), 283.

Oba, T., Kato, M., Kitazato, H., Koizumi, I., Omura, A., Sakai, T., and Takayama, T., in press. Paleoenvironmental changes in the Japan Sea during the last 85,000 years. Paleoceanography.

Pisias, N. G., and Leinen, M., 1984. Milankovitch forcing of the oceanic system: evidence from the Northwest Pacific. In Berger, A. L., Imbrie, J. Hays, G., Kukla, G., and Saltzman, B. (Eds.), Milankovitch and Climate (Pt. 1): Dordrecht (D. Reidel), 307-330.
Rea, D. K., and Janecek, T. R., 1982. Late Cenozoic changes in atmospheric circulation deduced from North Pacific eolian sediments. Mar. Geol., 49:149-167.

Ruddiman, W. F. and Janecek, T. R., 1989. Pliocene-Pleistocene biogenic and terrigenous fluxes at Equatorial Atlantic Sites 662, 663, and 664. In Ruddiman, W., Sarnthein, M., et al., Proc. ODP, Sci. Results, 108: College Station, TX (Ocean Drilling Program), 211-240.

Sarnthein, M., Tetzlaff, G., Koopmann, B., Wolter, K., and Pflaumann, U., 1981. Glacial and interglacial wind regimes over the eastern subtropical Atlantic and Northwest Africa. Nature, 293:193-196.

Sarnthein, M., Thiede, J., Pflaumann, U., Erlenkeuser, H., Fütterer, D., Koopmann, B., Lange, H., and Seibold, E., 1982. Atmospheric and oceanic circulation patterns off Northwest Africa during the past 25 million years. In von Rad, U., Hinz, K., Samthein, M., Seibold, E. (Eds.), Geology of the Northwest African Continental Margin: Berlin (Springer Verlag), 545-604.

Shackleton, N. J., Backman, J., Zimmerman, H., Kent, D. V., Hall, M. A., Roberts, D. G., Schnitker, D., Baldauf, J. G., Desprairies, A., Homrighausen, R., Huddlestun, P., Keene, J. B., Kaltenback, A. J., Krumsiek, K.A.O., Morton, A. C., Murray, J. W., and Westberg-Smith, J., 1984. Oxygen isotope calibration of the onset of ice-rafting and history of glaciation in the North Atlantic region. Nature, 37:620-623.

Stein, R., 1985a. Late Neogene changes of paleoclimate and paleoproductivity off Northwest Africa (DSDP Site 397). Palaeogeogr., Palaeoclimatol., Palaeoecol., 49:47-59.

1985b. The post-Eocene sediment record of DSDP Site 316: implications for African climate and plate tectonic drift. Mem.-Geol. Soc. Am., 163:305-315.

, 1991. Accumulation of organic carbon in marine sediments. Lect. Earth Sci., 34.

Tamaki, K., 1988. Geological structure of the Japan Sea and its tectonic implications. Chishitsu Chosasho Geppo, 39:269-365.

Thiede, J., 1979. Wind regimes over the late Quatenary southwest Pacific Ocean. Geology, 7:259-262.

Tungsheng, L., 1988. Loess in China: Stuttgart (Springer Verlag).

Ujiie, H., Ichikura, M., 1973. Holocene to uppermost Pleistocene planktonic foraminifers in a piston core from off the San'n district, Sea of Japan. Trans. Palaeontol. Soc. Jpn., New Ser., 9:137-150.

Van Andel, T. H., Heath, G. R., and Moore, T. C., 1975. Cenozoic history and paleoceanography of the Central Equatorial Pacific Ocean. Mem.-Geol. Soc. Am., 143.

Date of initial receipt: 22 May 1991

Date of acceptance: 27 September 1991

Ms 127/128B-145 